\title{
Interatomic and Intermolecular Coulombic Decay: The Early Years
}

\author{
U. Hergenhahn ${ }^{\mathrm{a}, 1}$ \\ ${ }^{a}$ Max-Planck-Institut für Plasmaphysik, Boltzmannstr. 2, 85748 Garching, Germany
}

\begin{abstract}
Autoionization is an important pathway for the relaxation of electronically excited states. In weakly bonded matter, efficient autoionization channels have been found, in which not only the initially excited state, but also neighbouring atoms or molecules take part. Since their theoretical prediction in 1997 these processes are known as Interatomic or Intermolecular Coulombic Decay (ICD). The author summarizes the experimental research on ICD up to the presence. Experiments on inner valence ICD in rare gas clusters, on cascade ICD after Auger decay and on ICD of satellite states are explicitly discussed. First experiments on water clusters and on solutes will be reviewed. An outlook on other non-local autoionization processes and on future directions of ICD research closes the article.
\end{abstract}

Key words: Intermolecular Coulombic Decay, ICD, Cluster, Autoionization 36.40.-c, 61.80.Fe, 82.50.-m

Email addresses: uwe.hergenhahn@ipp.mpg.de (U. Hergenhahn)

${ }^{1}$ Mail Address: IPP, c/o Helmholtz-Zentrum Berlin, Albert-Einstein-Str. 15, 12489 Berlin, Germany. 


\section{Introduction}

A vacancy site in an isolated atom or molecule can relax by flourescence, dissociation or - if energy permits - by autoionization. If we, instead of the isolated situation, consider a vacancy in a cluster of identical atoms or molecules, one may ask if and how the environment influences the relaxation process. In the case of a strong covalent bonding, such as in metal clusters, the electronic structure changes completely and any comparison would be difficult. In the case of weak bonding, e.g. by hydrogen bridges or dispersion forces, it is possible to discuss the electronic structure in terms of the one of the isolated system. It is this case which we will discuss here. Considering autoionization in particular, this can take place if the ionization energy used to produce the initial vacancy is above the double ionization threshold of the system. It has long been known that the double ionization threshold of clusters is lower with respect to the monomer [1]. This is natural, as in a cluster two hole states can have the vacancies located at different sites, resulting in a Coulomb repulsion energy which is lower than in the isolated system. But will these states play any role in autoionization, is it possible that a single vacancy in a weakly bonded cluster undergoes a direct transition into a state consisting of positive charges at two different sites and a continuum electron? In the last thirteen years it has been found that such autoionization channels indeed exist, that often they are far more effective than any other mode of relaxation and that they exhibit so many qualitative differences from other autoionization transitions that it is meaningful to designate them by a new name: Intermolecular or Interatomic Coulombic Decay (ICD), resp. [2], depending on whether we discuss a system composed of atomic or molecular entities.

This article intends to give a mini-review about the first years of research 
on ICD from a experimentalists perspective. The plan of the work is as follows: A simple example will be used as an introduction into the topic in the next subsection, followed by some essential points from the theory of ICD. A number of experiments will be reviewed next, separated into sections on noble gas clusters and on other systems. A variant of ICD taking place after resonant excitation, instead of non-resonant ionization, will be described after that. I will close with some remarks about the perspectives of the field, and will use an appendix to discuss relations between ICD and numerous other processes.

Due to the limited space available it is not possible to give a complete review of the field here, and I apologize to all whose important works are not cited here. Two useful reviews on the theory of ICD have appeared [3, 4].

\subsection{An example - the Ne dimer}

It is instructive to review an example. Figure 1 shows an energy diagram of Ne clusters in comparison to atomic Ne. Clearly, the Ne 2s level in atomic Ne cannot autoionize, and will decay by fluorescence on a ps time scale $[5,6]$. In a Ne dimer (or any larger Ne cluster) instead, a decay into a $\left(\mathrm{Ne}^{+} 2 \mathrm{p}^{-1}\right)_{2}$ two hole state is energetically viable. The first successful experiments on ICD in 2003 [7] and 2004 [8, 9] confirmed that the expected autoionization process indeed takes place. Figure 2 gives a sketch of the three steps involved in ICD of the Ne dimer initiated by photon impact: 1. Photoionization of an inner valence level, 2. autoionization (the actual ICD), 3. Coulomb explosion of the final state, as the two vacancies produced repell each other. The reaction equation in this system reads

$$
\begin{gathered}
h \nu+\mathrm{Ne}_{2} \rightarrow \\
\mathrm{NeNe}^{+}\left(2 \mathrm{~s}^{-1}\right)+e_{p h}^{-} \rightarrow
\end{gathered}
$$




$$
\mathrm{Ne}^{+}\left(2 \mathrm{p}^{-1}\right) \mathrm{Ne}^{+}\left(2 \mathrm{p}^{-1}\right)+e_{I C D}^{-}+e_{p h}^{-} .
$$

$\left(e_{p h}^{-}\right.$and $e_{I C D}^{-}$denote the photoelectron and the ICD electron, resp.) The signature of ICD has been seen in all three steps described above: A lifetime broadening of the photoelectron line resulting from the instability of the Ne $2 \mathrm{~s}$ level was demonstrated [9], the electrons resulting from ICD have been directly detected $[7,8]$ and the ion pair with opposite momenta of equal magnitude, created in the Coulomb explosion of the ICD final state, was seen using different variants of ion spectroscopy $[8,10]$.

\subsection{Theoretical Considerations}

Like all autoionization processes, ICD is driven by the Coulomb interaction between the electrons involved in the transition. The matrix element of the process can thus be written as

$$
\left\langle i v, \hat{\mathbf{k}} \varepsilon|V| o v, o v^{\prime}\right\rangle
$$

where $V$ is the Coulomb operator, $|i v\rangle$ is an inner valence electron, $|\hat{\mathbf{k}} \varepsilon\rangle$ the continuum orbital with momentum $\hat{\mathbf{k}}$ and energy $\varepsilon$, and $|o v\rangle,\left|o v^{\prime}\right\rangle$ outer valence orbitals located at one and the other site. It is important that for most cases the energy difference leading to IC decays is small. $|\hat{\mathbf{k}} \varepsilon\rangle$ describes an electron of low kinetic energy, that is of large wavelength. It is for this reason that the matrix element (2) may connect the two orbitals $|o v\rangle,\left|o v^{\prime}\right\rangle$ at different site effectively. For a more detailed discussion of the matrix element I refer to the literature $[3,11]$. Some results are mentioned here:

- The matrix element (2) factorizes into a direct and an exchange term, the former being associated with energy transfer between the two sites and the latter with charge transfer. It turns out that energy transfer in most systems dominates by far. Non-local autoionization going along with charge transfer will be discussed below (sec. 5). 
- The ICD rate depends strongly on the spatial distance $R$ between the two entities involved. Without considering overlap between the orbitals $|o v\rangle,\left|o v^{\prime}\right\rangle$ the rate drops $\sim R^{-6}$, characteristic of a dipole-dipole coupling. This is a remarkable property of ICD, as most energy and charge transfer processes known today have an exponential dependence on distance. In realistic cases however, finite overlap between the orbitals strongly modifies the ICD rate. That is saying, when $R$ is decreased from asymptotically large distances the rate increases much faster than $R^{-6}$ as overlap sets in [11]. The asymptotic case might be reached for the Ne dimer, but for most other systems discussed here it is probable that orbital overlap does have an influence on the rate of the decay.

- The $R^{-6}$ dependence of ICD is reminiscent of Förster Resonant Energy Transfer (RET) [12], another process driven by a dipole-dipole coupling. Intermolecular Coulombic Decay however is not a resonant process, and therefore is far more general as RET. As a dipole-dipole coupling in quantum electrodynamics is mediated by photon exchange, the exchange of a virtual photon has been used as a rationalization for the energy transfer going on in ICD. At least in this context there is no rigorous definition of the notion of a virtual photon. One may say it is shorthand for a certain matrix element resulting from Coulombic Interactions.

- Interatomic Coulombic Decay depends strongly on the number of nearest neighbours. The more neighbours, the faster ICD proceeds. For Ne, the rate saturates with $\mathrm{Ne}_{13}$, corresponding to one full shell of nearest neighbours [13]. 
The energetical prerequisites for ICD can be met in a very wide variety of systems, as has been recognized already in the first predictions of it [2]. It is therefore a phenomenon of universal importance, and recent experiments are starting to show this $[14,15,16]$.

Another way of expressing the physics in ICD is to say it is mediated by electron correlation. In theory, this view has enabled a most fascinating view on ICD: Using a formalism for the propagation of the correlated hole density in a quantum system, the authors of ref. [17] showed a time-dependent picture of the filling of a $\mathrm{Ne} 2 \mathrm{~s}$ vacancy from a $2 \mathrm{p}$ orbital in NeAr, and the synchronous creation of an Ar 3p vacancy and a continuum electron.

\section{Experiments on noble gas clusters}

The noble gas clusters were not the first systems, for which ICD was predicted [2]. From an experimental viewpoint they have the advantage of being prepared easily however. This is done by expanding the noble gas through a conical nozzle into vacuum [18]. In this process clusters can form via three-body collisions in the nozzle and subsequent aggregation $[19,20]$. It is well known that beams created such have a broad distribution of sizes $N$. The only value of $N$ for which an exclusive preparation can be achieved is the dimer, when the expansion is just driven at the onset of condensation [21].

Among the noble gas clusters, potential targets for the investigation of ICD are Ne clusters and mixed clusters of $\mathrm{Ne}$ and another noble gas. In Ar clusters, simple inner valence $\left(3 \mathrm{~s}^{-1}\right)$ vacancies are located below the double ionization threshold, but some satellite states can autoionize (see below). Interatomic Coulombic Decay is also expected as the second step in a cascade that starts with conventional Auger decay of a cluster. I will now expand on 
these three topics.

\subsection{ICD in Ne clusters}

The low kinetic energy part of the electron spectrum of photoionized Ne clusters was recorded by the author and coworkers in 2003 [7]. A feature was identified, which could not be observed in spectra of uncondensed $\mathrm{Ne}$ atoms nor in clusters irradiated with photon energies below the Ne 2s ionization threshold. It was correctly interpreted as resulting from ICD of the $2 \mathrm{~s}^{-1}$ level in Ne clusters. A pertinent spectrum from a somewhat later publication, covering both the ICD electrons and the 2s photoline [22], is shown in Fig. 3. In the latter work the area ratio of the two features was determined as a function of mean cluster size, and within the accuracy of the experiment showed a value of unity for the size range probed $(\langle N\rangle=50-500)$. These expirements were carried out with a conventional, hemispherical electron spectrometer to which a cluster jet was fitted [23]. Synchrotron radiation was used for the initial photoionization, same as in all experimental work discussed below. The low kinetic energy of the ICD electron, the low solid angle coverage of the analyzer, and the continuous background due to intracluster inelastical scattering entailed considerable experimental difficulty of these experiments. A lot of later works therefore used ion spectroscopy, electron-ion coincidence spectroscopy or electron-electron spectroscopy of some sort (see below).

Numerous works revealed further properties of ICD in Ne clusters. Jahnke et al., as mentioned above, used a COLTRIMS (Cold Target Recoil Ion Momentum Spectroscopy) spectrometer to detect in coincidence one of the electrons and both ions created by ICD of the Ne dimer [8]. In this apparatus, a static electric field is used to project the ionic fragments produced in a reaction onto a spatially and time resolving detector $[24,25]$. Using a pulsed excitation source, from the impact locations and times the three-dimensional 
momenta of the ions at the instant of production can be reconstructed. For the research on ICD, this method has been of great importance: Since the two positive charges in the final state of ICD repell each other, the detection of ion pairs with opposite momenta of equal magnitude is a sensitive indicator of the occurence of ICD. As there is no covalent bonding between the consituents of the system, a simple $1 / R$ law connects the Coulombic energy of the ion pair with distance. The sum of the ion kinetic energies can be inferred from the absolute values of the momenta if the fragment masses are known, e.g. in a dimer. This quantity is a measure of the internuclear distance at which ICD occured. It is termed kinetic energy release (KER). Using additionally an auxiliary magnetic field, slow electrons can be guided to a second detector opposite to the ion branch $[24,25]$.

Jahnke et al. demonstrated the Coulomb explosion of the dimer as a result of the decay, and showed that in this three body system the KER in the two ions mirrors the kinetic energy of the ICD electrons [8]. That is saying, total energy of the final state after ICD is a constant. In agreement with theory [26] the intensity in the electron spectrum vs. kinetic energy is maximal for values with almost vanishing kinetic energy. In the dimer, the repulsive potential curve of the final state, asymptotically two $\mathrm{Ne}^{+}$ground state ions, crosses the weakly bound potential curves of the inner valence singly ionized state at an internuclear distance somewhat lower than the ground state equilibrium [26]. The ICD energy spectrum is the $R$-dependent energy difference between the two states, which explains its observed shape (Fig. 4). In larger clusters the spectrum does not peak at $0 \mathrm{eV}$, but has a maximum between 1.2 and $1.6 \mathrm{eV}$ and drops towards lower energies again [7, 22, 27]. This difference is not rigorously explained yet, but can plausibly be attributed to final state polarization in extended clusters and to the suppression of Coulomb explosion 
due to the surroundings of the ion pair. The threshold regime of ICD in the Ne dimer is covered in [10], see below.

From the data set represented in [8] also the angular distribution of ICD electrons in the molecule fixed frame was extracted [28]. It was found to have a weak propensity for emission along the dimer axis. A theoretical study of NeAr also showed this trend [17].

Another work focussed on the lineshape of the Ne 2s photoline from large $(\langle N\rangle \approx 900)$ Ne clusters recorded in a conventional, hemispherical electron energy analyzer with a high energy resolution [9]. Generally, inner valence and core level photoelectron lines from medium-sized to large noble gas clusters are characterized by a two component structure resulting from different screening of bulk compared to surface initial states. Spectroscopically the splitting can be resolved easily [29]. In their study Öhrwall et al. established that both components in the case of Ne clusters have a Lorentzian lineshape, and determined the lifetime of bulk and surface states as $6 \pm 1 \mathrm{fs}$ and larger than $30 \mathrm{fs}$, resp. Exact spectroscopic data are also given and show that the $2 \mathrm{~s}$ related photoline is approx. $0.5 \mathrm{eV}$ wide, with a center binding energy around $48.1 \mathrm{eV}[9]$. This is compared to an atomic value of $48.475 \mathrm{eV}[30,31]$.

\section{2. $I C D$ in $N e$ Ar clusters}

Mixed noble gas clusters constitute another class of interesting prototype systems for the research on ICD. The morphology of mixed noble gas clusters has been investigated by photoelectron spectroscopy. For NeAr clusters created by expanding both gases simultaneously ('coexpansion') the growth of thin Ne films atop of an Ar core has been shown [32]. The reaction equation in this system reads

$$
h \nu+\mathrm{Ne}_{\mathrm{N}} \mathrm{Ar}_{\mathrm{M}} \rightarrow
$$




$$
\begin{gathered}
\mathrm{Ne}_{\mathrm{N}-1} \operatorname{Ar}_{\mathrm{M}} \mathrm{Ne}^{+}\left(2 \mathrm{~s}^{-1}\right)+e_{p h}^{-} \rightarrow \\
\mathrm{Ne}_{\mathrm{N}-1} \operatorname{Ar}_{\mathrm{M}-1} \mathrm{Ne}^{+}\left(2 \mathrm{p}^{-1}\right) \operatorname{Ar}^{+}\left(3 \mathrm{p}^{-1}\right)+e_{I C D}^{-}+e_{p h}^{-}
\end{gathered}
$$

As the ionization potential of Ar is lower than that of $\mathrm{Ne}$ (atomic values $15.76 \mathrm{eV}$ vs. $21.56 \mathrm{eV}[33,30])$, the ICD electron in this case will have a higher kinetic energy than in pure Ne clusters. A simple estimate using atomic binding energies, Coulombic repulsion and the equilibrium distances of the respective neutral clusters $(3.5 \AA$, [34]) gives $7 \mathrm{eV}$. In an experiment, the pertaining electrons were found at a somewhat higher energy of $8 \mathrm{eV}[35]$. The difference could be due to final state polarization in the experiment. Two further aspects of this work are of interest:

1. Argon condenses much earlier than neon, and whether in a coexpansion of neon and argon mixed clusters or pure argon clusters seeded by atomic Ne form, is a non-trivial question. In a more detailed study of this system the occurence of an ICD signal at the expected energy was used as a monitor for the condensation of Ne onto the clusters [32]. This demonstrated that the study of ICD has the promise to elucidate structural motifes of weakly bonded systems which might be difficult to obtain by other techniques [35]. This idea is illustrated by Fig. 5 .

2. From a theoretical viewpoint, the ICD spectrum of NeAr dimers has a richer structure than the one of pure $\mathrm{Ne}_{2}$. Neutral NeAr has two vibrationally excited states, the population of which leads to clearly measurable differences in the ICD spectrum [36]. This is because depending on the vibrational state the photoionization + ICD process occurs at different values of the internuclear distance $R$. Since the potential curve of the $\mathrm{Ne}^{+}+\mathrm{Ar}^{+}$final state is steeply repulsive, the nodal structure of the initial state reappears in the ICD spectrum. Somewhat unexpectedly this prediction was clearly observed in the spectra of Barth et al. although in their experiment clusters larger 
than the dimer were probed [35]. The finding agrees with other evidence for the population of surface states by Ne in mixed NeAr, as otherwise the Coulomb explosion in the final state would be hindered.

\subsection{ICD of satellite states}

In the examples I have described so far, a single-hole inner valence vacancy state undergoes autoionization. Besides these a large number of singly ionized states exist, which cannot be described as a single-hole configuration. The next more complicated class of singly ionized states is produced by the simultaneous ionization of one electron and a discrete excitation of another electron (two-hole one-particle states, 2h-1p). In the context of photoionization, lines pertaining to these states appear due to electron correlation and are called 'satellites'. In molecules the mixing between $1 h$ inner valence and $2 h-1 p$ states can be very strong ('breakdown of the molecular orbital picture'). The latter is saying that the binding energy of a lot of satellite states is similar to inner-valence ionization energies; in fact most satellites are slightly higher in energy. The question therefore arises whether these states can decay by ICD, too. A number of beautiful works consider this problem, and I will summarize three of them below:

\subsubsection{ICD of satellite states, Ar}

Contrary to $\mathrm{Ne}$, in an Ar cluster a $3 \mathrm{~s}^{-1}$ inner valence vacancy cannot undergo ICD, as the double ionization potential (DIP) with $32 \mathrm{eV}$ [37] even for larger clusters is too high by approx. $3.5 \mathrm{eV}$ [7, 29]. At binding energies above $32 \mathrm{eV}$ however several satellite states are located, the lower ones of which in an atomic language pertain to $3 \mathrm{p}^{4} 3 \mathrm{~d}, 3 \mathrm{p}^{4} 4 \mathrm{~s}$ and $3 \mathrm{p}^{4} 4 \mathrm{p}$ configurations. Lablanquie et al. probed a jet of Ar atoms and dimers in this range of excitation energies, and measured the yield and kinetic energy of the $\mathrm{Ar}^{+}$ 
cations being produced [38]. As the ionization of atomic Ar can only produce ions with vanishing kinetic energy (neglecting photoelectron recoil), energetic ions can be attributed to processes going on in the Ar dimer. The production of pairs of energetic cations is clearly observed at excitation energies above the DIP of an Ar dimer, when the KER of an $\mathrm{Ar}^{+}$ion pair produced at the dimer ground state geometry is added to this threshold (experimentally $34.85(5) \mathrm{eV})$. Numerous satellite states are located in this energy region. This finding is therefore interpreted as production of a dimer with an excited cation $\left(\operatorname{ArAr}^{+*}(S n)\right.$, with $(S n)$ designating some atomic satellite state) in a first step, and the subsequent autoionization by ICD in a second step.

It is interesting that cations with some kinetic energy $(0.75 \mathrm{eV})$ are observed at even lower photon energies, namely already above the Ar 3s ionization threshold (29.2 eV, [38]). The authors of Ref. [38] assigned them to the dissociation of $\operatorname{Ar}^{+*}\left(3 \mathrm{~s}^{-1}\right) \operatorname{Ar}$ into $\operatorname{Ar}^{+}\left(3 \mathrm{p}^{-1}\right)+\operatorname{Ar}^{*}\left(3 \mathrm{p}^{-1} 4 \mathrm{~s}\right)$. The latter ion + excited neutral pair at the dimer equilibrium geometry is formed at a point on its potential curve lying $0.75 \mathrm{eV}$ above the energetic minimum. The two step process consisting of 3s ionization followed by energy transfer to a neighbouring atom and dissociation was in fact observed in larger Ar clusters before the first experimental report on ICD, and is somewhat reminiscent to it, the difference being that the 'other' atom is not ionized [39].

Similar results on ICD of satellite states were also observed for $\mathrm{Kr}$ and Xe dimers [38].

\subsubsection{ICD of satellite states, $\mathrm{Ne}$}

A more subtle effect was observed in an extension of the data analysis of the experiment on the Ne dimer described above. Jahnke, Ueda et al. were able to also identify ion pairs pertaining to ICD of $2 p$ correlation satellites [40]. Several of these are apparent at binding energies a few eV higher than 
$\mathrm{Ne} 2 \mathrm{~s}$. In larger clusters they broaden as the excited electron changes its character from a Rydberg to an excitonic excitation [41], but in dimers their binding energies do not change much. We now consider explicitly the satellites in the binding energy interval $[50,58.5] \mathrm{eV}$. In an atomic language, all of these are characterized by a $2 \mathrm{p}^{4}$ core, to which some Rydberg electron is coupled. Interatomic Coulombic Decay would now proceed via a relaxation of the Rydberg electron into one of the $2 p$ vacancies, by which the binding energy difference to the first ionization potential is released, sufficient to ionize the neighbouring Ne atom. Indeed some configurations, e.g. $2 \mathrm{p}^{4}\left({ }^{1} D\right) 3 \mathrm{~s}$, decay by ICD at internuclear distances near to the neutral ground state, as observed from the KER to the ion pair. For other satellite configurations, e.g. $2 p^{4}\left({ }^{3} P\right) n p, n=3,4$ it turns out however that this simple type of decay is hindered. The reason is that a dipole transition, which is responsible for the energy transfer in ICD, cannot couple the $n \mathrm{p}$ electron to a $2 \mathrm{p}$ vacancy, since both single electron states are of equal parity. These satellites instead decay by an exchange-type matrix element

$$
\int \mathrm{d} \mathbf{r}_{1} \mathrm{~d} \mathbf{r}_{2} \psi_{\mathbf{k}}\left(\mathbf{r}_{1}\right) \phi_{i v}\left(\mathbf{r}_{2}\right) \frac{1}{\left|\mathbf{r}_{\mathbf{1}}-\mathbf{r}_{\mathbf{2}}\right|} \phi_{o v}\left(\mathbf{r}_{1}\right) \phi_{o v^{\prime}}\left(\mathbf{r}_{2}\right),
$$

where $\psi_{\mathbf{k}}$ denotes the continuum electron, $\phi_{i v}$ the inner valence orbital, and $\phi_{o v}, \phi_{o v^{\prime}}$ the outer valence orbitals at the site of the initial vacancy and the neighbouring site, resp. This matrix element involves a charge transfer (from $\phi_{o v^{\prime}}$ to $\left.\phi_{i v}\right)$ instead of an energy transfer. As this requires a spatial overlap of the wavefunctions, the magnitude of the matrix element depends exponentially on the internuclear distance, and not just by a power law. The decay can only proceed after the internuclear separation has reduced substantially. As the equilibrium geometry of the noble gas dimers ions however is substantially contracted with respect to the neutral ground state, nuclear dynamics will proceed such as to enable the decay. The lower internuclear distance at 
the moment of ICD is reflected in a larger KER, which was the experimental fact that gave rise to the above interpretation.

\subsubsection{ICD of satellite states, He}

An extreme example for ICD has been observed in the decay of satellite states in the He dimer. After photoionization into the $n=2$ or higher satellites (configuration of the dimer is $\mathrm{He} \mathrm{He}^{+}(n l)$ ), ICD is viable from an energetical viewpoint. The ground state of the He dimer is extremely loosely bound though, with $\langle R\rangle=52 \AA[42]$. The Coulomb explosion characteristic for ICD nevertheless has been observed [43]. A theoretical model again highlights the decisive role of nuclear dynamics in the decay [44].

\subsection{ICD after Auger decay}

In ICD as discussed so far, a singly ionized state in a cluster decays into a non-local two-hole state. An analogous situation can arise when a doubly ionized state, situated on a single constitutent of a weakly bonded system, energetically is placed above the threshold for creating a triply ionized state, which involves a double and a single vacancy at two different sites. This type of ICD can occur in a cascade after conventional (inner shell) Auger decay in a cluster [45]. In Ar clusters for example, the part of the Auger spectrum which involves 3 s vacancies after the first decay can decay further to states of the type $\operatorname{Ar}^{2+}\left(3 \mathrm{p}^{-2}\right) \mathrm{Ar}^{+}\left(3 \mathrm{p}^{-1}\right)$. Similar conditions prevail for other noble gas clusters, and are predicted for clusters of simple molecules. For the latter experimental data are missing however.

\subsubsection{ICD after Auger decay, Ar}

For noble gas clusters on the other hand Ueda and coworkers have published a series of COLTRIMS experiments, in which they have investigated the decay of high-lying doubly ionized states that are populated by Auger 
decay. The first results have been obtained on Ar dimers. To understand this work it is helpful to briefly review the normal $L_{2,3} M M$ Auger spectrum of atomic $\mathrm{Ar}[46]$. In the lower range of kinetic energies (175-195 eV), three prominent doublets of Auger lines are visible. The doublet splitting is consistent with population of the same final state from either the $2 \mathrm{p}_{3 / 2}$ or the $2 \mathrm{p}_{1 / 2}$ vacancy. All of these final states receive their intensity from Auger decay into the $3 \mathrm{~s}^{-1} 3 \mathrm{p}^{-1}$ configuration, which is split into three states however due to mixing with the $3 \mathrm{~s}^{2} 3 \mathrm{p}^{-3} 3 \mathrm{~d}$ satellite. The least energetic doublet of Auger lines, at 177.9 and $180.1 \mathrm{eV}$ kinetic energy, is populating a doubly charged atomic state, which in a dimer with a neutral partner is situated above the triple ionization threshold of the dimer. The atomic triple ionization threshold is at even higher energies though. In an experiment by Morishita et al. [47] one of the electrons emitted in the following reaction

$$
\begin{array}{r}
h \nu+\operatorname{Ar}_{2} \rightarrow \operatorname{Ar}^{+}\left(2 \mathrm{p}^{-1}\right) \mathrm{Ar}+e_{p h}^{-} \rightarrow \\
\operatorname{Ar}^{2+}\left[3 \mathrm{p}^{-3} 3 \mathrm{~d}(48 \%)+3 \mathrm{~s}^{-1} 3 \mathrm{p}^{-1}(37 \%)\right] \mathrm{Ar}+e_{a u}^{-}+e_{p h}^{-} \rightarrow \\
\operatorname{Ar}^{2+}\left(3 \mathrm{p}^{-2}\right) \operatorname{Ar}^{+}\left(3 \mathrm{p}^{-1}\right)+e_{I C D}^{-}+e_{a u}^{-}+e_{p h}^{-}
\end{array}
$$

was detected in coincidence with both the $\mathrm{Ar}^{2+}$ and the $\mathrm{Ar}^{+}$cations. $\left(e_{a u}^{-}\right.$ denotes the Auger electron.) From the KER it could be shown that the production of this ion pair takes place at an internuclear distance of $3.7 \AA$, very close to the equilibrium distance of the $\operatorname{Ar}$ dimer (3.8 $\AA$ ). The kinetic energy of the electron fitted to either an Ar 2p photoelectron, or an ICD electron with an energy estimated from the respective binding energy differences and the Coulomb repulsion of the ions. Remeasured data with improved statistics revealed more detail on this reaction [48]. In this reference, next to the reaction (5) some less intense ICD channels populated from Auger final states with higher binding energy can be seen.

A full discussion of these results is more complex than in the case of inner 
valence ICD of singly ionized states, since numerous other decay pathways are feasible after $2 \mathrm{p}$ ionization. It needs a thorough discussion to show that the signature observed in Ref.s $[47,48]$ cannot be produced in any other reaction than (5). A comprehensive treatment of all relevant processes has been presented by Stoychev et al. [49], based on ab initio calculations of a large number of Ar dimer potential curves. I would like to pick out two aspects: 1. Lacking evidence for non-local amplitudes in the inner shell $\left(L_{2,3} M M\right)$ decay of the $\mathrm{Ar}$ dimer and 2. alternative pathways ending up in an $\left(\mathrm{Ar}^{+}\right.$, $\left.\mathrm{Ar}^{+}\right)$, instead of $\left(\mathrm{Ar}^{2+}, \mathrm{Ar}^{+}\right)$, ion pair.

1. Non-local amplitudes in inner shell Auger decay have been discussed $[50,51]$, and evidence exists that they are important for molecules with strongly electronegative ligands $[52,53]$ (see below). Although $\mathrm{Ar}_{2}$ is not of that type it is important to rule out Auger decay to $\mathrm{Ar}^{+*} \mathrm{Ar}^{+}$, followed by atomic autoionization, as an alternative pathway to $\left(\mathrm{Ar}^{2+}, \mathrm{Ar}^{+}\right)$. In Ref. [49] only one channel is identified for which both steps of the process are energetically viable. It would lead to production of the final ion pair at larger internuclear distance, that is with smaller KER, than experimentally observed (see [48]).

2. The strong abundance of $\left(\mathrm{Ar}^{+}, \mathrm{Ar}^{+}\right)$pairs in the data shown in Ref.s [47, 48] again raises the question for non-local Auger amplitudes. Further experimental data for these final states have been discussed by Saito et al. [54]. These authors analyzed the energy of the Auger electron pertaining to the ion pair, and found that the latter arrives in coincidence with electrons from all parts of the $L_{2,3} M M$ spectrum. The KER, also presented in [48], indicates a break-up predominantly at an internuclear distance smaller than the neutral equilibrium. As the potential curves of the dimer after local decay into the most intense $\operatorname{Ar}^{2+}\left(3 \mathrm{p}^{-2}\right) \mathrm{Ar}$ states have minima 
around those values, it has been concluded that the nuclear wavepacket from the ground state evolves towards lower $R$, where a (slow) radiative decay, $\operatorname{Ar}^{2+}\left(3 \mathrm{p}^{-2}\right) \operatorname{Ar} \rightarrow \operatorname{Ar}^{+}\left(3 \mathrm{p}^{-1}\right) \operatorname{Ar}^{+}\left(3 \mathrm{p}^{-1}\right)+h \nu$, takes place.

Some results on ICD of the Ar trimer are discussed in Ref.s [55, 56].

Studies on cascade ICD after inner shell Auger decay were also successful in ArKr [57] after $\operatorname{Ar} L_{2,3} M M$ Auger decay and in $\mathrm{Kr}_{2}$ after $\mathrm{Kr} M_{4,5} N N$ Auger decay [58].

It is worthwhile to mention a point of interdisciplinary interest in these cascade decays: The importance of slow electrons for dissociation of biomolecules has been revealed in the last years [59, 60, 61]. Although it is clear that slow electrons are the most abundant product after absorption of any type of energetic radiation in living tissue, the models about their production and thermalization are still rather schematic. Interatomic or molecular Coulombic Decay is one such source of slow electrons. At the same time it produces not one but two positively charged vacancies, which may lead to alterations of the nuclear structure at the same point where a slow electron is produced.

\subsubsection{ICD after Auger decay, $\mathrm{Ne}$}

After these spectroscopic experiments succeeded, ICD following inner shell Auger decay was utilized to shed light on the quantum mechanical nature of inner shell vacancies: In a homonuclear diatomic molecule, the dichotomy between left - right and gerade-ungerade core hole states has fascinated researchers for quite some decades, and always with the advent of new experimental methods new arguments in favour of one or the other interpretation were carved. In ethyne $\left(\mathrm{C}_{2} \mathrm{H}_{2}\right)[62]$ and somewhat later in nitrogen [63] the energy difference between $g$ and $u$ 1s vacancy states was spectroscopically observed. In a 'molecule' like $\mathrm{Ne}_{2}$ an analogous experiment would be 
difficult, as the ratio between the $g \mid u$ splitting and the lifetime broadening would be far less favourable. Other experiments can be constructed however in an attempt to observe in retrospect whether a 1s vacancy has behaved in a localized or delocalized manner $[64,65]$. In the Ne dimer, the 1s shell was singly ionized by synchrotron radiation. After relaxation by $K L L$ Auger decay - assumed to proceed in a quasiatomic fashion - some states underwent a Coulomb explosion leading to an energetic $\mathrm{Ne}^{+}+\mathrm{Ne}^{2+}$ ion pair, obviously by an Auger-ICD cascade decay. The vector of fragment relative motion also yielded the orientation of the molecular axis at the instant of emission of the ICD electron. Since ICD is a fast process it is safe to assume that it coincides with the molecular axis direction in the moment of photoionizaton. In two experiments the angular distribution of the photoelectrons relative to the direction of the fragment momenta was observed [64,65]. For a delocalized (gerade or ungerade) core hole, this angular distribution should preserve inversion symmetry with respect to the molecular center, but not for a localized core hole. In both experiments, a broken symmetry with respect to the molecular center was found, most clearly for a $\mathrm{Ne}_{2}$ ensemble aligned along the electric field vector of the ionizing radiation, but more subtle also for molecules with an axis direction $\perp$ to the electric field. The experiments were therefore interpreted as having proven experimentally the localization of the initial core hole in $\mathrm{Ne}_{2}[64,65]$. One could call this expected, as due to the observable distinction in the charge state of the final Ne atoms this part of the final state ensemble is projected on the $l$ or $r$ eigenstates of the dimer system. (Examining the photoelectron data in closer detail reveals a statistically significant disagreement between the two independent experiments as to the exact shape of the molecule-fixed 1s angular distribution function. While the experiments in Ref. [64] and two independent, theoretical data 
sets in Ref.s $[64,65]$ show a propensity of electron emission towards the $\mathrm{Ne}^{+}$ fragment, this trend is not displayed by the experimental data of Ref. [65].)

Kreidi et al. also derived the angular distribution of the ICD electrons in the molecule fixed frame [64]. Again, these angular distribution function are asymmetric with respect to inversion at the molecular center, and moreover different between ICD proceeding via dipole-dipole coupling vs. ICD proceeding via an exchange-type matrix element (see 2.3.2).

Considerable further detail resulted from the experimental work of Kreidi et al. In a full paper following their initial publication they were able to characterize all pathways by which a Ne dimer can relax after single photon core level photoionization slightly above the threshold [66]. Again, the quantitatively most important channel is breakup into $\mathrm{Ne}^{+}+\mathrm{Ne}^{+}$after radiative decay of single-site doubly charged vacancy states populated by Auger decay (mostly $2 \mathrm{p}^{4}\left({ }^{1} D\right)$ and $2 \mathrm{p}^{4}\left({ }^{1} S\right)$ ). For the asymmetric break-up into $\mathrm{Ne}^{+}+\mathrm{Ne}^{2+}, \mathrm{ICD}$ with the reaction equation

$$
\begin{array}{r}
h \nu+\mathrm{Ne}_{2} \rightarrow \mathrm{Ne}^{+}\left(1 \mathrm{~s}^{-1}\right) \mathrm{Ne}+e_{p h}^{-} \rightarrow \\
\mathrm{Ne}^{2+}\left(2 \mathrm{~s}^{-1} 2 \mathrm{p}^{-11} P\right) \mathrm{Ne}+e_{a u}^{-}+e_{p h}^{-} \rightarrow \\
\mathrm{Ne}^{2+}\left(2 \mathrm{p}^{-21} D\right) \mathrm{Ne}^{+}\left(2 \mathrm{p}^{-12} P\right)+e_{I C D}^{-}+e_{a u}^{-}+e_{p h}^{-}
\end{array}
$$

was corroborated. Besides that ICD mediated by exchange matrix elements $\left(\mathrm{ICD}_{E T}\right)$, as in (2.3.2), could be isolated. Some of the observed channels can occur by either $\left(\operatorname{ICD}_{E T}\right)$ or ETMD (see sec. 5), but no experimental separation was possible in these cases. Another, minor, contribution to both symmmetric and asymmetric break-up are channels in which charge transits from one to the other dimer atom via crossings of the respective potential curves. In the same work, also ICD after $2 \mathrm{~s}$ photoionization is revisited. It would have been impossible to arrive at this comprehensive picture of the process without the fruitful collaboration with theory, see Stoychev et al. 
[67].

In an improved variant of this experiment the authors tested their spectra for fingerprints of the wavepacket dynamics in the Ne dimer during ICD [68]. We have discussed that the KER spectrum includes the competition with dynamics in an integral fashion, but in Ref. [68] the authors went beyond that by measuring the electron kinetic energy spectrum as a function of emission angle relative to the momentum of the doubly charged fragment. Thus, one should be able to see the influence of wavepacket motion in the dimer on the ICD spectrum, which according to theory is considerable [68, 69]. This wavepacket motion is initiated by recoil from either the Auger electron (ICD after Auger decay) or the 2s photoelectron (ICD after 2s ionization at high photon energy). Experimentally, energy differences pointed in the same direction as predicted, but with a much smaller magnitude.

\section{Experiments on water and solvents}

The experiments described so far all had one thing in common: They were carried out on noble gas clusters. These are prototypical for weakly bonded systems. Intermolecular Coulombic Decay however should prevail with other types of weak bonding. The initial predictions e.g. considered $\mathrm{HF}$ and $\mathrm{H}_{2} \mathrm{O}$ clusters $[2,70,71]$. The search for ICD in molecular clusters turned out to be more tedious than thought. One obvious difference to noble gas clusters is the larger density of final states. While ICD may lead to a rather confined spectral line in noble gas clusters, in molecular clusters it smears out to a quasi-continuum, even when nuclear dynamics is not influential. In clusters larger than a few units, inelastic electron scattering is a competition in the creation of low kinetic energy electrons, which leads to a rather structureless background the area of which scales with the clusters size and the oscillator 
strength for outer valence ionization. Simple electron spectroscopy, such as in e.g. Ref.s [7, 35], therefore so far did not deliver an unambiguous result. The argument concerning the final states can be easily seen from theoretical work, e.g. on the simulated ICD spectra of $\left(\mathrm{H}_{2} \mathrm{O}\right)_{2}$ to $\left(\mathrm{H}_{2} \mathrm{O}\right)_{4}[72]$.

\subsection{ICD in water clusters}

Eventually, two experiments on ICD in water clusters were successful $[16,15]$. Both used coincidence detection techniques. When ICD is initiated by photoionization, the energy of the primary electron (the photoelectron) is well known. In an experiment which is capable of detecting two electrons in coincidence, it is therefore possible to set a filter to primary electrons of this kinetic energy to selectively detect only secondary electrons, which had followed inner valence ionization of a selected level.

If only the vertical ionization potentials are considered, the energetics for inner valence ICD in water cluster is not much different from noble gas clusters: Molecular water is known to have one rather broad, featureless inner valence line (final state $2 \mathrm{a}_{1}^{-1}$ ) at a (vertical) binding energy of $32.3 \mathrm{eV}$ [73]. (Earlier experiments gave $32.2 \mathrm{eV}$ [74] and $32.6 \mathrm{eV}$ [75].) This energy is known to shift to somewhat lower values in clusters $(32.0 \mathrm{eV}$ for $\langle N\rangle=$ 100, [76]) and in liquid water $(30.9(1) \mathrm{eV},[77])$. Calculations of the double ionization potentials of water clusters in a neutral ground state geometry have been presented for sizes up to $N=4$ [72]. For $N=4$ for example, they start at $26.28 \mathrm{eV}$ for two-site double vacancy states. Again, the single-site double vacancy states with energies of $37.98 \mathrm{eV}$ and higher are above the inner valence ionization energy.

To ascertain that autoionization of inner valence states in water is only viable by ICD, and not by molecular processes, it is necessary to also discuss the adiabatic double ionization threshold. The sum of the ionization energies 
for a separated $\mathrm{OH}+\mathrm{H}$ radical pair is only $26.6 \mathrm{eV}$ [78], and by threshold electron coincidence spectroscopy [79] an onset of photo-double-ionization of molecular water at a photon energy of $31.6 \mathrm{eV}$ has been seen. This is only possible by single photoionization followed by dissociation of the molecule (at least to some extent), and subsequent autoionization [79, 80]. For final state energies lower than $34.4 \mathrm{eV}$ however, double ionization so far has only been seen at the respective threshold, and not at higher photon energies [80, 16]. That is saying, while energetically molecular autoionization of $2 \mathrm{a}_{1}^{-1}$ states is allowed, after a vertical ionization process such as photoionization the respective channels seem to be closed and autoionization without assistance by the environment is hindered.

\subsubsection{Investigating ICD by electron, electron coincidence techniques}

To isolate a signature of ICD in water clusters was challenging, as explained above. It turned out to be essential to simultaneously detect both electrons created in an ICD reaction in coincidence and with high collection efficiency. In an experiment carried out in the group of the author, a magnetic bottle spectrometer was used for that purpose [81, 82, 27]. Here, an inhomogenuous magnetic field is used to sample even electrons with fairly high kinetic energies $(>100 \mathrm{eV})$ from almost $4 \pi$ sR solid angle. At the same time, the magnetic field is reliably guiding electrons of kinetic energies down to $100 \mathrm{meV}$ and lower to the detector. One can then do a targeted experiment on the secondary electron spectrum attributed to inner valence photoionization (only) of water clusters. First of all, a significant yield of slow electrons with a kinetic energy distribution independent from the primary electron is expected. It is this fact which distinguishes photoionization + ICD from direct photo-double-ionization and from sequential double ionization by intra-cluster electron scattering. In the two latter processes, the 
two electrons being emitted share all of the available phase space, which is getting larger with the available excess energy (photon energy reduced by two hole final state energy).

It is helpful to use a well-known system to introduce the characteristic features of electron, electron coincidence spectra of clusters. In Fig. 6 results for Ne clusters are shown. This system has been discussed above (2.1) and we have seen an isolated ICD feature at kinetic energies of 1.2-1.6 eV (Fig. 3). This ICD line shows up again in the lower third of the colour-coded electron pair intensity map, with a kinetic energy of the faster electron $\left(e_{1}\right)$ corresponding to the $\mathrm{Ne} 2 \mathrm{~s}$ photoline, and with the slower electron $\left(e_{2}\right)$ energy as seen before. The profile of the 2s line can be seen when the coincident signal is summed up along all $e_{2}$ energies (panel c). Here, no monomer signal is visible as the uncondensed part of the beam does not lead to electron, electron coincidences. The ICD energy spectrum can be extracted by summing up the signal along $e_{1}$ energies, but only in the region where a primary electron pertaining to ICD autoionization is involved (red bars).

Competing double ionization processes may show up in maps like panel b of Fig. 6. In the valence region, electron impact ionization by intracluster inelastic scattering is the main competitor. This process can have a considerable intensity in larger clusters [83, 27]. Different than in ICD, any energy sharing between the two electrons is kinematically possible. Propensity rules, which often favour an unequal sharing of energies, are not influential in the valence region. Electron impact ionization therefore will lead to a homogenuously populated stripe of intensity along a line of constant total energy, being at a right angle to the diagonal of Fig. 6, panel b. The minimum allowed total energy equals twice the ionization energy of the monomer. In clusters as small as $\langle N\rangle=45$ this cannot be realized: Electron impact ionization is 
faintly seen as a diagonal ending at $8 \mathrm{eV}$ total kinetic energy, corresponding to the Coulomb repulsion at a distance of approx. $10 \AA .^{2}$

Using the magnetic bottle spectrometer for this experiment not only adds the capability to record the process in coincidence, but also shows that in larger Ne clusters, different than in the dimer, the ICD intensity drops towards zero energy. Since the hemispherical electron analyzer used in earlier experiments $[7,22]$ had a strongly decreasing transmission function for electrons below one $\mathrm{eV}$ in kinetic energy, this was impossible to show at that time.

\subsubsection{ICD in medium sized water clusters}

Electron pairs having the ICD signature were indeed found in electronelectron coincidence spectra of free water cluster jets [16] (Fig. 7). Mean cluster sizes of 45 and 200 molecules were investigated. Several cross checks can be made to underpin the validity of the interpretation given: When a jet consisting of uncondensed molecules is probed, the coincidence feature due to ICD vanishes. Instead, the two double ionized final states of molecular water, as identified firstly by John Eland [80], are seen in the final state spectrum. From the coincident electron intensity, the kinetic energy spectrum of the ICD electrons can be determined by integration over all photoelectron energies in the inner valence range. The result is a rather unstructured spectrum which is ascending towards very low electron energies (Fig. 7, a) and in qualitative agreement with the predictions for small clusters [84]. It is interesting that even for very low energies no local maximum is seen, and in this respect water is different from Ne clusters. The presence of inelastic

\footnotetext{
${ }^{2}$ In Fig. 6 some narrow striations from bottom left to top right are also visible. These result from an electronics artefact.
} 
electron scattering occuring in competition serves as another check of the experimental method. Electron pairs with a fixed total energy, appearing as diagonal lines in the coincidence plot, are indeed seen in high contrast plots of the coincident intensities.

\subsubsection{ICD in water dimers}

In water dimers, a COLTRIMS experiment on ICD was equally successful [15]. By choosing clusters of the smallest conceivable size, a lot of problems due to a background of scattered secondary electrons can be avoided. These authors recorded coincident events of two singly charged water cations with opposite momenta, and of two electrons (four particles were detected in coincidence). First of all, seeing pairs of water cations already is a hint of some exceptionally fast creation mechanism, as in other experiments on water cluster ionization always protonated fragments of the type $\left(\mathrm{H}_{2} \mathrm{O}\right)_{n} \mathrm{H}^{+}$were observed (e.g. $[85,86,87])$. This is explained by the formation of ion cores $\left(\mathrm{H}_{3} \mathrm{O}^{+}\right.$or $\left.\mathrm{H}_{5} \mathrm{O}_{2}^{+}\right)$and the release of an $\mathrm{OH}$ radical in the course of that. At least in theory this reaction proceeds within tens of fs [88, 89], and therefore is one of the fastest known processes involving nuclear processes. Any reasonable candidate for a reaction that is foreclosing this channel should therefore invoke the electronic structure only to create the two distributed vacancies. (As the experiment was carried out with synchrotron radiation, which is produced in the form of ps long pulses of low intensity, any two-photon processes in the ionization could be ruled out.) The energy distribution of the electrons received is at least qualitatively consistent with a combination of a water inner valence photoelectron line with an ICD spectrum in the shape shown in Ref. [16]. A KER of approx. $4.2 \mathrm{eV}$ has been measured for the two water cations. This is slightly lower than expected from the ground state oxygenoxygen distance of the water dimer $(2.9 \AA$, [90]). Meanwhile, the Coulomb 
explosion of the dimer has been simulated and the discrepancy could be explained from an unusual amount of rotational energy acquired by the ions during the dissociation [90].

\subsection{ICD in solutions}

It is an exciting perspective to employ the $R^{-6}$ distance dependence and the substance dependence of the ICD spectrum to investigate solvent geometries. A suitable technique to probe solutions by electron spectroscopy, pioneered by Faubel and Winter [91], uses a liquid jet injected under high pressure into vacuum. Two studies relevant to ICD were carried out by this technique. The first such work considered the deexcitation of resonantly excited $\mathrm{OH}^{-}$ions in water [92], and will be discussed below (4.1). Another recent study presents a thorough investigation of the decay of $L$-shell vacancies in potassium and cloride, solvated in water [14].

In solution with water, $\mathrm{KCl}$ dissociates into $\mathrm{K}^{+}$and $\mathrm{Cl}^{-}$ions. $L_{2,3}$ vacancies were produced in these ions by synchrotron radiation, and the experimental decay spectra were interpreted by ab initio calculations. For the case of $\mathrm{K}^{+}$, it was found that Auger decay channels - transitions from $\mathrm{K}^{2+}\left(2 \mathrm{p}^{-1} 4 \mathrm{~s}^{-1}\right)$ to a $\mathrm{K}^{3+}$ state with the outermost $4 \mathrm{~s}$ and two $3 \mathrm{p}$ valence electrons removed - are by far the dominating feature in the spectrum. Besides these however ICD channels, populating states with vacancies located on both the potassium ion and the surrounding water molecules, were observed. For a system consisting of a $\mathrm{K}^{+}$decorated with some $\mathrm{H}_{2} \mathrm{O}$ molecules, decay spectra were calculated, the above mentioned mixed vacancy states were shown to be significantly different in final state energy, and also in theory they are populated with an intensity of some $\%$ with respect to the main Auger channels. The calculation of final state energies corroborates the assignment of the experimental feature identified with ICD-like channels. In 
$\mathrm{Cl}^{-}$, the situation is more difficult as the ICD-like channels overlap in energy with the Auger channels. Although calculations predicted both channels to be present, this could not be asserted or disproven from the experiment.

\section{Resonant ICD}

Soon after the discovery of ICD, discussion of a resonant variant of this process started. In this so-called resonant ICD, an inner valence vacancy is produced not by ionization but by excitation into some unoccupied orbital. First experiments were done at about the same time by Barth et al. on large Ne clusters [93] and by Aoto et al. on the Ne dimer [10]. Barth et al. clearly saw the production of an ICD like slow electron peak at two excitation energies below the cluster 2 s ionization threshold. The energies compared well to resonant excitations found in earlier experiments on thick condensed Ne layers.

In the dimer experiment of Aoto et al. the broadening mechanisms found in beams of larger clusters are absent. As in [38], spectroscopy of energetic $\mathrm{Ne}^{+}$ions and ion pairs was used. Numerous resonances below and above the atomic $2 \mathrm{~s}$ threshold could be singled out and were identified as final states of normal ICD or, below threshold, as final states of a resonant variant of ICD in which $\mathrm{Ne} 2 \mathrm{~s}^{-1} n l \mathrm{Ne}$ undergoes spectator decay to $\mathrm{Ne} 2 \mathrm{p}^{-1} n l \mathrm{Ne}^{+} 2 \mathrm{p}^{-1}$.

A theoretical account on resonant ICD has also been given, this time about $\mathrm{MgNe}$ after Ne 2s excitation [94]. After Ne 2s excitation, autoionization into a local single hole state $\mathrm{Mg}\left(\mathrm{Ne}^{+} 2 \mathrm{p}^{-1}\right)$ is the most probable channel in that system, but resonant ICD receives an intensity in the same order of magnitude. 


\subsection{Resonant ICD in solutions}

So far, I have discussed ICD after inner valence excitaton, which is following the original conceptual work. If we consider transitions at higher energies, as a rule a decay into a local two-hole state is viable. A general discussion of Interatomic or Intermolecular transition amplitudes vs. Auger decay is deferred to a later point, but here I would like to present results of a first experiment that may indicate ICD-like behaviour in the deexcitation of an inner-shell vacancy of $\mathrm{OH}^{-}$solvated in water [92]. It was done by electron spectroscopy on a liquid jet, as described above [91]. The authors tuned the excitation energy of a synchrotron radiation beam to a resonant core excitation of the $\mathrm{O}$ 1s orbital in $\mathrm{OH}^{-}, 1 \mathrm{~s} \rightarrow$ CTTS. (CTTS stands for charge transfer to solvent, see [92]). Other 1s resonances, e.g. of the solvent water, are energetically separated. The deexcitation spectrum of the $\mathrm{OH}^{-}$ $\mathrm{O}$ 1s vacancy shows three features specific to the resonance. Their binding energies can trivially be determined, as there is only one outgoing electron. According to the authors, these states are too low in binding energy to be explained with local 2h-1p configurations from spectator resonant Auger decay of the $\mathrm{OH}^{-*}$ state. Moreover, from the electronic configuration of $\mathrm{OH}^{-}$ [95] it is not obvious how a splitting into three states could occur. An alternative, striking explanation has been found: The final states of the features in question could be constructed from one vacancy in the $\mathrm{OH}^{-}$and another one in a valence orbital of the surrounding water solvent shell, which is ionized by an ICD-like energy transfer. This explanation fits quite well to the observed energies in the deexcitation spectrum. It was further supposed that this energy transfer greatly gains in efficiency by orbital overlap between the $\mathrm{OH}^{-}$and the solvent shell. This finding has very important implications as it may help to decide between contradicting proposals for solution mechanisms 
of the hydroxide $\mathrm{OH}^{-}$ion.

\section{Other non-local autoionization processes}

Other autoionization schemes in loosely bound complexes were proposed from theory besides ICD. Again, they are characterized by a final state consisting of two vacancies distributed to two units forming the aggregate. Autoionization processes, in which the initial vacancy is not filled locally, but by electron transfer from a neighbouring atom or molecule, have been discussed using the term Electron Transfer Mediated Decay (ETMD) [96]. (More precisely, ETMD differs from ICD in the final charge state of the site that contained the initial vacancy: In ETMD it ends up with one less unit of charge than before the decay, e.g. a singly charged vacancy after the decay is neutral.) One can differentiate between $\operatorname{ETMD}(2)$, involving one neighbouring site that becomes doubly charged, and $\operatorname{ETMD}(3)$ (see Fig. 8), which involves two neighbouring atoms $[96,53,84]$. Instead of an energy transfer, as in ICD, ETMD involves a charge transfer between two sites. Theoretically it was found that the transition amplitudes for this type of decay are orders of magnitude lower than those involving energy transfer $[96,84]$. This is in line with experimental results on non-local autoionization of satellite channels, for which ICD vs. energy transfer is ruled out by selection rules [40]. Electron Transfer Mediated Decay can therefore not compete with ICD in systems in which both channels are open. In heterogeneous systems however a situation might occur where ETMD is the only viable radiationless decay channel, and then it may become observable. Examples identified theoretically are Ar 3s vacancies in small ArKr and ArXe clusters [97, 98], and experiments on Kr core-Ar shell systems have yielded experimental evidence for ETMD [99].

Electron Transfer Mediated Decay has also been mentioned as an ex- 
planation for the ionic fragment spectra of larger ArXe mixed clusters [100]. These experiments called for an efficient mechanism for the transfer of charge from Ar ionized states to neighbouring Xe sites. ETMD might be one such mechanism.

Another class of systems, in which ETMD was considered theoretically, are $\left(\mathrm{H}_{2} \mathrm{O}\right)_{2} \mathrm{Li}^{+}$clusters. After Li 1s ionization no electrons are remaining at the Li which could fill the vacancy locally and ETMD for this reason is the only viable autoionization channel [101].

In ICD and ETMD the energy required in the autoionization step is afforded by relaxation at some site that has initially been ionized by photoionization, or (in future experiments possibly) by electron impact. Alternatively, one can consider a process in which an extended system captures a slow electron from the continuum. The energy gained such is transferred to another site of the system by electron correlation, which is ionized in that way. This process is competing to radiative recapture, in which the excess energy is radiated away by a photon. Two model systems have been identified up to now, in which this so-called interatomic Coulombic electron capture (ICEC) can become effective, and it is expected to be a phenomenon of general importance [102]. One example is a $\mathrm{Mg}^{2+}$ center decorated with a water solvent shell: When an electron, even with a very low kinetic energy, is captured by the $\mathrm{Mg}$ dication, the energy gained such is sufficient to ionize one of the neighbouring water molecules. This will result in a Coulomb explosion of the complex, just like in ICD.

\section{Perspectives of the field}

Experiments so far made on ICD fall mainly into one of two groups: Those using conventional electron spectroscopy on larger clusters and liquids 
or those using electron spectroscopy in coincidence with momentum resolved ion detection on dimer systems. The latter have revealed precise information on the energy and dynamics of ICD in small systems, while the former have shown the relevance and the application potential of ICD. Most recently, electron-electron coincidence spectroscopy on larger clusters has added to that list. What comes next? To close the discussion, I would like to point out some perspectives of research on non-local autoionization phenomena.

Certainly, we are just beginning to explore the chemical diversity of ICD. Predictions of this phenomenon have been made for solvent complexes [101, 103], endohedral fullerenes [104], alkaline-noble gas compounds [94, 105] and doped He droplets [106].

New developments in the field of light sources for the VUV spectral range have enabled the creation of ultrashort pulses (attosecond range), and the investigation of autoionization phenomena in the time domain [107]. Application of these techniques to ICD has the potential to yield a much deeper understanding of the decay mechanism (see [17]), in particular with respect to a competition with nuclear dynamics.

Coming to implications of this phenomena, the potential role of slow electrons for radiation damage has been mentioned (2.4.1). Certainly, a lot of different processes can occur when an energetic particle interacts with living matter, ICD being only one of them. From radiation biology it is known that cell damage due to ionizing radiation occurs when a DNA strand is broken at two adjacent positions (double strand breaks) [108], or is damaged in a more complex way [109]. How these lesions are produced on a molecular level we are just beginning to understand [110, 111]. As ICD produces two cations plus a low energy electron that may induce further processes via dissociative attachment $[59,60,61]$ it might have an important role in this 
context. Certainly, more and interdisciplinary research is needed here.

Experiments on NeAr clusters (2.2) point out to the potential importance of ICD to research on interfaces, e.g. in weakly bonded systems. The application of ICD to research on solvent chemistry has been mentioned $(3.2,4.1)$. Another - yet visionary - application might be in solar cells: It is currently proposed that the efficiency of solar cells could be strongly increased if the absorption would occur in a nano-crystalline material [112]. One current problem is the transfer of energy from the light absorbing nano-crystals to the substrate. Radiationless energy transfer processes have been proposed in this context [113].

Another area of current interest, much more fundamtental in nature, is strongly ionized matter produced in the focus of new, ultra-intense radiation sources in the VUV and X-ray range, so-called Free Electron Lasers. New autoionization channels are predicted to occur in this regime, which become possible because a large number of the constituents of the system can be transferred into an excited or ionized state at one and the same time [114, $115]$.

\section{Acknowledgements}

The author would like to acknowledge all current and past group members who have worked with him on ICD experiments (S. Marburger, O. Kugeler, S. Barth, M. Mucke, M. Förstel, T. Arion, V. Ulrich, T. Lischke, S. Joshi). Discussions with L. S. Cederbaum, N. Sisourat and with R. Dörner are gratefully acknowledged. This work has been partially funded by the Deutsche Forschungsgemeinschaft. 


\section{Appendix}

Energy transfer and autoionization occur in a plethora of systems in physics and chemistry, and numerous other processes have aspects which invite a comparison with Interatomic or Intermolecular Coulombic Decay.

Auger decay is an extremely well known autoionization process of, typically, inner shell vacancies in atoms, molecules and bulk condensed matter. A characteristic of Auger decay is the relatively high transition energy (several ten to several thousand $\mathrm{eV}$ ). The continuum state is therefore unable to couple several ionic sites in the transition matrix element (2) and the spectrum is determined by local transitions. Different to that most ICD processes discussed in this article occur at excitation energies, for which a decay to a local two-hole state energetically is not viable. The different nature of the final states in Auger decay can also be seen from the fact that, in molecules, they often are metastable and only dissociate on a much longer time scale (e.g. $[116,117])$.

For bulk condensed matter, a classical paper on the relation between bandwidth, Coulombic repulsion and localization in Auger spectra is by Sawatzky [118]. Again, one sees two regimes both different from ICD: Either the final state has an atomic character or the final state is delocalized over the whole valence band, which requires strong overlap between the orbitals at neighbouring sites of the crystal.

Auger decay and non-local autoionization processes discussed in this review meet in the case of molecules with strongly elecronegative ligands. An example that was investigated experimentally is the $\mathrm{Si} L_{2,3} V V$ decay in $\mathrm{SiF}_{4}$ [52]. When an inner shell in the $\mathrm{Si}$ core is ionized, it has been observed that the lifetime broadening is much larger than expected from a purely local model for Auger decay. As the strongly electronegative fluorine ligands are 
pulling away charge from the Si center, such model predicts a longer lifetime of $\mathrm{Si}$ core holes in $\mathrm{SiF}_{4}$ compared to other $\mathrm{Si}$ compounds. The opposite is the case. The decrease in core hole lifetime was interpreted by the occurence of non-local decay amplitudes in an Auger process (Thomas et al., [52]).

In an attempt to rationalize these findings Buth et al. have systematically calculated energies and orbital character of the two-hole states in the xenon fluorides (Xe and $\mathrm{XeF}_{N}$, with $N=2,4,6$ ) [53]. A population analysis of these states showed the increasing importance of fluorine vacancies for dicationic states in $\mathrm{XeF}_{4,6}$. In a second step the character of the Auger transition rates for filling a Xe 4d vacancy was analyzed. All transition amplitudes were expanded into a set of atomic basis functions, and thus expressed as some 'transition strength' (basically the square of the atomic decay matrix element) times the respective population numbers. Each term in this expansion can be grouped into one of the four categories local decay, ICD, $\operatorname{EMTD}(2)$ and $\operatorname{ETMD}(3)$. Using the further assumption that the transition strengths are different between each category, but identical for all individual transitions within one category, it was possible to arrive at the relative importance of each type of transition. An impressive trend showed up: Already in $\mathrm{XeF}_{2}$ ICD-like amplitudes clearly dominate over the local ones, and are in $\mathrm{XeF}_{4}$ and $\mathrm{XeF}_{6}$ even superseded by $\operatorname{ETMD}(3)$ [53]. The main factor underlying these findings is the nature of the strongly electronegative ligands; in most other molecules, Auger decay is a mainly local process as stated above.

Multi-atom resonant photoemission (MARPE) is another process which raised the hope of learning about nearest neighbour relationships from electron spectroscopy [51]. Here, in bulk metal oxides an influence of core level resonances in one atom to Auger emission from the other atom was found. These transitions occur at energies of several hundred eV, and local ampli- 
tudes should be dominant. Indeed, the effect is small but significant. It currently is described on a microscopic level as a one-step resonant scattering process, or in a macroscopic picture as a frequency-dependent change of the constituents dielectric function. In the one-step description, formally the same matrix element as in ICD appears, although clad in a KramersHeisenberg picture.

Charge exchange and energy transfer are central processes also in the diverse field of collision physics. Two representants that are vaguely reminiscent to ICD and/or ETMD are Penning ionization-ionization of gas phase or condensed targets by energy transfer from metastable He ions [119] — and 'Auger neutralization', which is an electron emission process occuring after the impact of slow ionic projectiles onto bulk surfaces $[120,121]$. Both of these processes are topics of intense research in their own right, and the references given here are just examples picked from a vast literature. While they have in common to ICD (ETMD) that energy (and/or charge) is transferred between two systems not chemically bound to each other, there are also significant differences. As these are impact processes, transitions occur over a range of relative positions of the two interacting systems, and this makes a more succinct comparison difficult. On the other hand, research on ICD/ETMD is developing rapidly, and it seems quite conceivable that this and other fields, such as the ones just mentioned, will in future mutually benefit from each other.

\section{References}

[1] P. Scheier, A. Stamatovic, T. D. Märk, J. Chem. Phys. 88 (1988) 42894293. 
[2] L. S. Cederbaum, J. Zobeley, F. Tarantelli, Phys. Rev. Lett. 79 (1997) $4778-4781$.

[3] R. Santra, L. S. Cederbaum, Phys. Rep. 368 (2002) 1-117.

[4] V. Averbukh, P. V. Demekhin, P. Kolorenc, S. Scheit, S. D. Stoychev, A. I. Kuleff, Y.-C. Chiang, K. Gokhberg, S. Kopelke, N. Sisourat, L. S. Cederbaum, J. Electron Spectrosc. Relat. Phenom (2010) DOI: 10.1016/j.elspec.2010.03.003.

[5] K.-H. Schartner, B. Magel, B. Möbus, H. Schmoranzer, M. Wildberger, J. Phys. B 23 (1990) L527.

[6] C. T. Johnson, A. E. Kingston, Journal of Physics B: Atomic and Molecular Physics 20 (1987) 5663.

[7] S. Marburger, O. Kugeler, U. Hergenhahn, T. Möller, Phys. Rev. Lett. 90 (2003) 203401.

[8] T. Jahnke, A. Czasch, M. S. Schöffler, S. Schössler, A. Knapp, M. Käsz, J. Titze, C. Wimmer, K. Kreidi, R. E. Grisenti, A. Staudte, O. Jagutzki, U. Hergenhahn, H. Schmidt-Böcking, R. Dörner, Phys. Rev. Lett. 93 (2004) 163401.

[9] G. Öhrwall, M. Tchaplyguine, M. Lundwall, R. Feifel, H. Bergersen, T. Rander, A. Lindblad, J. Schulz, S. Peredkov, S. Barth, S. Marburger, U. Hergenhahn, S. Svensson, O. Björneholm, Phys. Rev. Lett. 93 (2004) 173401.

[10] T. Aoto, K. Ito, Y. Hikosaka, E. Shigemasa, F. Penent, P. Lablanquie, Phys. Rev. Lett. 97 (2006) 243401-4. 
[11] V. Averbukh, I. B. Müller, L. S. Cederbaum, Phys. Rev. Lett. 93 (2004) 263002-4.

[12] G. D. Scholes, Annu. Rev. Phys. Chem. 54 (2003) 57-87.

[13] R. Santra, J. Zobeley, L. S. Cederbaum, Phys. Rev. B 64 (2001) 245104.

[14] W. Pokapanich, H. Bergersen, I. L. Bradeanu, R. R. T. Marinho, A. Lindblad, S. Legendre, A. Rosso, S. Svensson, O. Björneholm, M. Tchaplyguine, G. Öhrwall, N. V. Kryzhevoi, L. S. Cederbaum, J. Am. Chem. Soc. 131 (2009) 7264-7271.

[15] T. Jahnke, H. Sann, T. Havermeier, K. Kreidi, C. Stuck, M. Meckel, M. Schöffler, N. Neumann, R. Wallauer, S. Voss, A. Czasch, O. Jagutzki, A. Malakzadeh, F. Afaneh, T. Weber, H. SchmidtBöcking, R. Dörner, Nature Physics 6 (2010) 139-142.

[16] M. Mucke, M. Braune, S. Barth, M. Förstel, T. Lischke, V. Ulrich, T. Arion, U. Becker, A. Bradshaw, U. Hergenhahn, Nature Physics 6 (2010) 143-146.

[17] A. I. Kuleff, L. S. Cederbaum, Phys. Rev. Lett. 98 (2007) 083201-4.

[18] O. F. Hagena, Rev. Sci. Instrum. 63 (1992) 2374-2379.

[19] R. Karnbach, M. Joppien, J. Stapelfeldt, J. Wörmer, T. Möller, Rev. Sci. Instrum. 64 (1993) 2838-2849.

[20] D. R. Miller, Oxford University Press, 1988.

[21] P. M. Dehmer, S. T. Pratt, J. Chem. Phys. 76 (1982) 843-853.

[22] S. Barth, S. Marburger, O. Kugeler, V. Ulrich, S. Joshi, A. M. Bradshaw, U. Hergenhahn, Chem. Phys. 329 (2006) 246-250. 
[23] S. P. Marburger, O. Kugeler, U. Hergenhahn, AIP Conference Proceedings 705 (2004) 1114-1117.

[24] R. Dörner, V. Mergel, O. Jagutzki, L. Spielberger, J. Ullrich, R. Moshammer, H. Schmidt-Böcking, Phys. Rep. 330 (2000) 95-192.

[25] J. Ullrich, R. Moshammer, A. Dorn, R. Dörner, L. P. H. Schmidt, H. Schmidt-Böcking, Rep. Prog. Phys. 66 (2003) 1463-1545.

[26] S. Scheit, V. Averbukh, H.-D. Meyer, N. Moiseyev, R. Santra, T. Sommerfeld, J. Zobeley, L. S. Cederbaum, J. Chem. Phys. 121 (2004) 83938398.

[27] M. Mucke, U. Hergenhahn, et al, to be published.

[28] T. Jahnke, A. Czasch, M. Schöffler, S. Schössler, M. Käsz, J. Titze, K. Kreidi, R. E. Grisenti, A. Staudte, O. Jagutzki, L. P. H. Schmidt, S. K. Semenov, N. A. Cherepkov, H. Schmidt-Böcking, R. Dörner, J. Phys. B 40 (2007) 2597.

[29] R. Feifel, M. Tchaplyguine, G. Öhrwall, M. Salonen, M. Lundwall, R. R. T. Marinho, M. Gisselbrecht, S. L. Sorensen, A. Naves de Brito, L. Karlsson, N. Mårtensson, S. Svensson, O. Björneholm, Eur. Phys. J. D 30 (2004) 343-351.

[30] K. Harth, M. Raab, H. Hotop, Z. Phys. D 7 (1987) 213-25.

[31] W. Persson, Physica Scripta 3 (1971) 133-155.

[32] M. Lundwall, W. Pokapanich, H. Bergersen, A. Lindblad, T. Rander, G. Öhrwall, M. Tchaplyguine, S. Barth, U. Hergenhahn, S. Svensson, O. Björneholm, J. Chem. Phys. 126 (2007) 214706-8. 
[33] I. Velchev, W. Hogervorst, W. Ubachs, J. Phys. B 32 (1999) L511.

[34] S. M. Cybulski, R. R. Toczylowski, J. Chem. Phys. 111 (1999) 1052010528.

[35] S. Barth, S. P. Marburger, S. Joshi, V. Ulrich, O. Kugeler, U. Hergenhahn, Phys. Chem. Chem. Phys. 8 (2006) 3218-3222.

[36] S. Scheit, V. Averbukh, H.-D. Meyer, J. Zobeley, L. S. Cederbaum, J. Chem. Phys. 124 (2006) 154305-8.

[37] E. Rühl, C. Schmale, H. C. Schmelz, H. Baumgärtel, Chem. Phys. Lett. 191 (1992) 430-434.

[38] P. Lablanquie, T. Aoto, Y. Hikosaka, Y. Morioka, F. Penent, K. Ito, J. Chem. Phys. 127 (2007) 154323-9.

[39] R. Thissen, P. Lablanquie, R. I. Hall, M. Ukai, K. Ito, Eur. Phys. J. D 4 (1998) 335-342.

[40] T. Jahnke, A. Czasch, M. Schöffler, S. Schössler, M. Käsz, J. Titze, K. Kreidi, R. E. Grisenti, A. Staudte, O. Jagutzki, L. P. H. Schmidt, T. Weber, H. Schmidt-Böcking, K. Ueda, R. Dörner, Phys. Rev. Lett. 99 (2007) 153401-4.

[41] S. Joshi, S. Barth, S. Marburger, V. Ulrich, U. Hergenhahn, Phys. Rev. B 73 (2006) 235404-7.

[42] R. E. Grisenti, W. Schöllkopf, J. P. Toennies, G. C. Hegerfeldt, T. Köhler, M. Stoll, Phys. Rev. Lett. 85 (2000) 2284-2287.

[43] T. Havermeier, T. Jahnke, K. Kreidi, R. Wallauer, S. Voss, M. Schöffler, S. Schössler, L. Foucar, N. Neumann, J. Titze, H. Sann, M. Kühnel, 
J. Voigtsberger, J. H. Morilla, W. Schöllkopf, H. Schmidt-Böcking, R. E. Grisenti, R. Dörner, Phys. Rev. Lett. 104 (2010) 133401.

[44] N. Sisourat, N. V. Kryzhevoi, P. Kolorenc, S. Scheit, T. Jahnke, L. S. Cederbaum, Nature Physics 6 (2010) 508-511.

[45] R. Santra, L. S. Cederbaum, Phys. Rev. Lett. 90 (2003) 153401.

[46] H. Pulkkinen, S. Aksela, O.-P. Sairanen, A. Hiltunen, H. Aksela, J. Phys. B 29 (1996) 3033-3050.

[47] Y. Morishita, X.-J. Liu, N. Saito, T. Lischke, M. Kato, G. Prümper, M. Oura, H. Yamaoka, Y. Tamenori, I. H. Suzuki, K. Ueda, Phys. Rev. Lett. 96 (2006) 243402-4.

[48] K. Ueda, X.-J. Liu, G. Prümper, H. Fukuzawa, Y. Morishita, N. Saito, J. Electron Spectrosc. Relat. Phenom. 155 (2007) 113-118.

[49] S. D. Stoychev, A. I. Kuleff, F. Tarantelli, L. S. Cederbaum, J. Chem. Phys. 128 (2008) 014307.

[50] T. X. Carroll, J. Hahne, T. D. Thomas, L. J. Sæthre, N. Berrah, J. Bozek, E. Kukk, Phys. Rev. A 61 (2000) 042503.

[51] A. W. Kay, F. J. Garcia de Abajo, S.-H. Yang, E. Arenholz, B. S. Mun, N. Mannella, Z. Hussain, M. A. Van Hove, C. S. Fadley, Phys. Rev. B 63 (2001) 115119.

[52] T. D. Thomas, C. Miron, K. Wiesner, P. Morin, T. X. Carroll, L. J. Sæthre, Phys. Rev. Lett. 89 (2002) 223001.

[53] C. Buth, R. Santra, L. S. Cederbaum, J. Chem. Phys. 119 (2003) $10575-10584$. 
[54] N. Saito, Y. Morishita, I. Suzuki, S. Stoychev, A. Kuleff, L. Cederbaum, X.-J. Liu, H. Fukuzawa, G. Prümper, K. Ueda, Chem. Phys. Lett. 441 (2007) 16-19.

[55] N. Saito, X. J. Liu, Y. Morishita, I. H. Suzuki, K. Ueda, J. Electron Spectrosc. Relat. Phenom. 156-158 (2007) 68-72.

[56] X.-J. Liu, N. Saito, H. Fukuzawa, Y. Morishita, S. Stoychev, A. Kuleff, I. H. Suzuki, Y. Tamenori, R. Richter, G. Prümper, K. Ueda, Journal of Physics B: Atomic, Molecular and Optical Physics 40 (2007) F1.

[57] Y. Morishita, N. Saito, I. H. Suzuki, H. Fukuzawa, X.-J. Liu, K. Sakai, G. Prümper, K. Ueda, H. Iwayama, K. Nagaya, M. Yao, K. Kreidi, M. Schöffler, T. Jahnke, S. Schössler, R. Dörner, T. Weber, J. Harries, Y. Tamenori, J. Phys. B 41 (2008) 025101.

[58] K. Ueda, H. Fukuzawa, X.-J. Liu, K. Sakai, G. Prümper, Y. Morishita, N. Saito, I. Suzuki, K. Nagaya, H. Iwayama, M. Yao, K. Kreidi, M. Schöffler, T. Jahnke, S. Schössler, R. Dörner, T. Weber, J. Harries, Y. Tamenori, J. Electron Spectrosc. Relat. Phenom. 166-167 (2008) $3-10$.

[59] B. Boudaiffa, P. Cloutier, D. Hunting, M. A. Huels, L. Sanche, Science 287 (2000) 1658-1660.

[60] I. Bald, J. Kopyra, I. Dabkowska, E. Antonsson, E. Illenberger, J. Chem. Phys. 126 (2007) 074308.

[61] E. Brun, P. Cloutier, C. Sicard-Roselli, M. Fromm, L. Sanche, J. Phys. Chem. B 113 (2009) 10008-10013. 
[62] B. Kempgens, H. Köppel, A. Kivimäki, M. Neeb, L. S. Cederbaum, A. M. Bradshaw, Phys. Rev. Lett. 79 (1997) 3617-20.

[63] U. Hergenhahn, O. Kugeler, A. Rüdel, E. E. Rennie, A. M. Bradshaw, J. Phys. Chem. A 105 (2001) 5704-5708.

[64] K. Kreidi, T. Jahnke, T. Weber, T. Havermeier, R. E. Grisenti, X. Liu, Y. Morisita, S. Schössler, L. P. H. Schmidt, M. Schöffler, M. Odenweller, N. Neumann, L. Foucar, J. Titze, B. Ulrich, F. Sturm, C. Stuck, R. Wallauer, S. Voss, I. Lauter, H. K. Kim, M. Rudloff, H. Fukuzawa, G. Prümper, N. Saito, K. Ueda, A. Czasch, O. Jagutzki, H. SchmidtBöcking, S. K. Semenov, N. A. Cherepkov, R. Dörner, J. Phys. B 41 (2008) 101002.

[65] M. Yamazaki, J.-i. Adachi, Y. Kimura, A. Yagishita, M. Stener, P. Decleva, N. Kosugi, H. Iwayama, K. Nagaya, M. Yao, Phys. Rev. Lett. 101 (2008) 043004-4.

[66] K. Kreidi, T. Jahnke, T. Weber, T. Havermeier, X. Liu, Y. Morisita, S. Schössler, L. P. H. Schmidt, M. Schöffler, M. Odenweller, N. Neumann, L. Foucar, J. Titze, B. Ulrich, F. Sturm, C. Stuck, R. Wallauer, S. Voss, I. Lauter, H. K. Kim, M. Rudloff, H. Fukuzawa, G. Prümper, N. Saito, K. Ueda, A. Czasch, O. Jagutzki, H. Schmidt-Böcking, S. Stoychev, P. V. Demekhin, R. Dörner, Phys. Rev. A 78 (2008) 043422 .

[67] S. D. Stoychev, A. I. Kuleff, F. Tarantelli, L. S. Cederbaum, J. Chem. Phys. 129 (2008) 074307.

[68] K. Kreidi, P. V. Demekhin, T. Jahnke, T. Weber, T. Havermeier, X.-J. Liu, Y. Morisita, S. Schössler, L. P. H. Schmidt, M. Schöffler, M. Oden- 
weller, N. Neumann, L. Foucar, J. Titze, B. Ulrich, F. Sturm, C. Stuck, R. Wallauer, S. Voss, I. Lauter, H. K. Kim, M. Rudloff, H. Fukuzawa, G. Prümper, N. Saito, K. Ueda, A. Czasch, O. Jagutzki, H. SchmidtBöcking, S. Scheit, L. S. Cederbaum, R. Dörner, Phys. Rev. Lett. 103 (2009) 033001.

[69] P. V. Demekhin, S. Scheit, S. D. Stoychev, L. S. Cederbaum, Phys. Rev. A 78 (2008) 043421.

[70] J. Zobeley, L. S. Cederbaum, F. Tarantelli, J. Phys. Chem. A 103 (1999) $11145-11160$.

[71] J. Zobeley, L. S. Cederbaum, F. Tarantelli, J. Chem. Phys. 108 (1998) 9737-9750.

[72] I. B. Müller, L. S. Cederbaum, J. Chem. Phys. 125 (2006) 204305-12.

[73] S. Y. Truong, A. J. Yencha, A. M. Juarez, S. J. Cavanagh, P. Bolognesi, G. C. King, Chem. Phys. 355 (2009) 183-193.

[74] A. W. Potts, W. C. Price, Proceedings of the Royal Society A 326 (1972) 181.

[75] M. S. Banna, B. H. McQuaide, R. Malutzki, V. Schmidt, J. Chem. Phys. 84 (1986) 4739-4744.

[76] S. Barth, M. Oncak, V. Ulrich, M. Mucke, T. Lischke, P. Slavicek, U. Hergenhahn, J. Phys. Chem. A 113 (2009) 13519-13527.

[77] B. Winter, R. Weber, W. Widdra, M. Dittmar, M. Faubel, I. Hertel, J. Phys. Chem. A 108 (2004) 2625-2632. 
[78] J. D. Barr, A. de Fanis, J. M. Dyke, S. D. Gamblin, N. Hooper, A. Morris, S. Stranges, J. B. West, T. G. Wright, J. Chem. Phys. 110 (1999) $345-354$.

[79] S. Y. Truong, A. J. Yencha, A. M. Juarez, S. J. Cavanagh, P. Bolognesi, G. C. King, Chem. Phys. Lett. 474 (2009) 41-44.

[80] J. H. Eland, Chem. Phys. 323 (2006) 391-396.

[81] P. Kruit, F. H. Read, J. Phys. E 16 (1983) 313-324.

[82] P. Lablanquie, L. Andric, J. Palaudoux, U. Becker, M. Braune, J. Viefhaus, J. Eland, F. Penent, J. Electron Spectrosc. Relat. Phenom. 156-158 (2007) 51-57.

[83] V. Ulrich, Untersuchung von Autoionisationsprozessen in kleinen Molekülen und Clustern mittels hochaufösender Elektronenkoinzidenzspektroskopie, Ph.D. thesis, Technische Universität Berlin, 2007.

[84] I. B. Müller, L. S. Cederbaum, J. Chem. Phys. 122 (2005) 094305.

[85] V. Hermann, B. D. Kay, A. W. Castleman, Chem. Phys. 72 (1982) $185-200$

[86] H. Shiromaru, H. Shinohara, N. Washida, H.-S. Yoo, K. Kimura, Chem. Phys. Lett. 141 (1987) 7-11.

[87] P. P. Radi, P. Beaud, D. Franzke, H.-M. Frey, T. Gerber, B. Mischler, A.-P. Tzannis, J. Chem. Phys. 111 (1999) 512-518.

[88] H. Tachikawa, J. Phys. Chem. A 108 (2004) 7853-7862.

[89] A. Furuhama, M. Dupuis, K. Hirao, J. Chem. Phys. 124 (2006) 16431010. 
[90] O. Vendrell, S. D. Stoychev, L. S. Cederbaum, ChemPhysChem 11 (2010) 1006-1009.

[91] B. Winter, M. Faubel, Chem. Rev. 106 (2006) 1176-1211.

[92] E. F. Aziz, N. Ottosson, M. Faubel, I. V. Hertel, B. Winter, Nature 455 (2008) 89-91.

[93] S. Barth, S. Joshi, S. Marburger, V. Ulrich, A. Lindblad, G. Öhrwall, O. Björneholm, U. Hergenhahn, J. Chem. Phys. 122 (2005) 241102.

[94] K. Gokhberg, V. Averbukh, L. S. Cederbaum, J. Chem. Phys. 124 (2006) 144315-9.

[95] B. Winter, M. Faubel, I. Hertel, C. Pettenkofer, S. Bradforth, B. Jagoda-Cwiklik, L. Cwiklik, P. Jungwirth, J. Am. Chem. Soc. 128 (2006) 3864-3865.

[96] J. Zobeley, R. Santra, L. S. Cederbaum, J. Chem. Phys. 115 (2001) $5076-5088$.

[97] M. Pernpointner, N. V. Kryzhevoi, S. Urbaczek, The Journal of Chemical Physics 129 (2008) 024304.

[98] E. Fasshauer, N. V. Kryzhevoi, M. Pernpointner, The Journal of Chemical Physics 133 (2010) 014303.

[99] M. Förstel, U. Hergenhahn, et al, to be published. This conference.

[100] M. Hoener, D. Rolles, A. Aguilar, R. C. Bilodeau, D. Esteves, P. O. Velasco, Z. D. Pešić, E. Red, N. Berrah, Phys. Rev. A 81 (2010) 021201.

[101] I. B. Müller, L. S. Cederbaum, J. Chem. Phys. 122 (2005) 094305-11. 
[102] K. Gokhberg, L. S. Cederbaum, J. Phys. B 42 (2009) 231001.

[103] I. B. Müller, L. S. Cederbaum, J. Phys. Chem. A 108 (2004) 5831-5844.

[104] V. Averbukh, L. S. Cederbaum, Phys. Rev. Lett. 96 (2006) 053401-4.

[105] V. Averbukh, L. S. Cederbaum, J. Chem. Phys. 123 (2005) 204107.

[106] N. V. Kryzhevoi, V. Averbukh, L. S. Cederbaum, Phys. Rev. B 76 (2007) 094513.

[107] M. Drescher, M. Hentschel, R. Kienberger, M. Uiberacker, V. Yakovlev, A. Scrinzi, T. Westerwalbesloh, U. Kleineberg, U. Heinzmann, F. Krausz, Nature 419 (2002) 803-807.

[108] J. S. Bedford, W. C. Dewey, Radiation Research 158 (2002) 251-291.

[109] N. Shikazono, M. Noguchi, K. Fujii, A. Urushibara, A. Yokoya, Journal of Radiation Research 50 (2009) 27-36.

[110] M. A. Huels, B. Boudaïffa, P. Cloutier, D. Hunting, L. Sanche, Journal of the American Chemical Society 125 (2003) 4467-4477.

[111] A. Eschenbrenner, M. A. H. D. Penhoat, A. Boissiere, G. Eot-Houllier, F. Abel, M. F. Politis, A. Touati, E. Sage, A. Chetioui, Int. J. Radiat. Biol. 83 (2007) 687-697.

[112] W. A. Tisdale, K. J. Williams, B. A. Timp, D. J. Norris, E. S. Aydil, X.-Y. Zhu, Science 328 (2010) 1543-1547.

[113] S. Chanyawadee, R. T. Harley, D. Taylor, M. Henini, A. S. Susha, A. L. Rogach, P. G. Lagoudakis, Applied Physics Letters 94 (2009) 233502.

[114] V. Averbukh, P. Kolorenč, Phys. Rev. Lett. 103 (2009) 183001. 
[115] A. I. Kuleff, K. Gokhberg, S. Kopelke, L. S. Cederbaum, Phys. Rev. Lett. 105 (2010) 043004.

[116] D. Schröder, H. Schwarz, J. Phys. Chem. A 103 (1999) 7385-7394.

[117] R. Püttner, X.-J. Liu, H. Fukuzawa, T. Tanaka, M. Hoshino, H. Tanaka, J. Harries, Y. Tamenori, V. Carravetta, K. Ueda, Chem. Phys. Lett. 445 (2007) 6-11.

[118] G. A. Sawatzky, Phys. Rev. Lett. 39 (1977) 504-507.

[119] H. T. Schmidt, S. H. Schwartz, A. Fardi, K. Haghighat, H. Cederquist, L. Liljeby, A. Langereis, J. C. Levin, I. A. Sellin, Phys. Rev. A 58 (1998) 2887-2894.

[120] H. D. Hagstrum, Phys. Rev. 96 (1954) 336-365.

[121] H. Brongersma, M. Draxler, M. de Ridder, P. Bauer, Surface Science Reports 62 (2007) 63-109.

[122] S. Barth, Untersuchung des Interatomaren Coulomb-Zerfalls in schwach gebundenen Systemen, Phd, Technical University Berlin, 2007. 


\section{Figure Captions}

Figure 1: Sketch of the energy levels relevant for inner valence ICD in a Ne atom (panel a) compared to Ne clusters (panel b). In a cluster, the $2 \mathrm{~s}^{-1}$ inner valence vacancy can autoionize into states with two vacancies at two different, preferentially neighouring sites (arrow pointing downward). Exact ionization energies depend on the cluster size and - in larger clusters - on the ionized site (see text). For an atom, only atomic doubly ionized state are available for autoionization, which are located at higher energies however. Autoionization transitions from inner valence singly ionized states into the former are therefore energetically not possible (arrows pointing upward).

Figure 2: Sketch of Interatomic Coulombic Decay in a Ne dimer. a) The $2 \mathrm{~s}$ valence level is ionized by a photon. b) A $2 \mathrm{p}$ electron relaxes into the vacancy. The energy released by that is transferred to the neighbouring atom via a virtual photon (see text). Theoretical work shows that these two processes indeed occur at the same time. c) Two atomic ions with outer valence vacancies have been formed. As the system has been bonded very weakly, their potential curve is plainly repulsive. A Coulomb explosion follows. From [8], Copyright: American Physical Society.

Figure 3: Electron kinetic energy distribution after photoionization of a free Ne cluster jet. The contribution of uncondensed monomers has been subtracted, and the kinetic energy dependence of the analyzer transmission has been corrected. Two prominent lines due to Ne 2s photoionization and due to ICD of this vacancy are visible. The minor lines between the two features are due to $2 p$ correlation satellites [41]. From [22], Copyright: Elsevier. 
Figure 4: Qualitative view of the potential curves relevant for an ICD experiment on a neutral rare gas dimer. $E_{\text {exc }}$ is the energy difference to the ground state. The neutral ground state is very weakly bound with a large equilibrium $R$. Ionization results in a cationic state with a stronger binding, as the positive charge can polarize the other atom (top-most curve). The energy difference between the two states is not drawn to scale. From there, autoionization (ICD) can occur and results in a plainly repulsive state final state. The energy difference leading to ICD normally is small, a few eV at most. Nuclear dynamics in the singly ionized state can compete with ICD. See [4] and references therein.

Figure 5: Photoelectron spectrum of large, mixed NeAr clusters (panels c, d) and ICD spectrum for decay into $\mathrm{Ne}^{+} \mathrm{Ar}^{+}$final states (panels a, b) $[35,122,32]$. Black symbols show the measured data while continuous lines result from least squares fits. The top row $(\mathrm{a}, \mathrm{c})$ shows mixed clusters that are rich in Ar, while clusters in the bottom row $(b, d)$ are rich in Ne. The relative Ar content determined by photoemission is shown in the figure $[122,32]$, with the Ar content of the gas mixture before expansion given in brackets. Ne photoemission spectra for clusters with high Ar content show only one component, assigned to atoms in surface states on a compact $\mathrm{Ar}$ core. For clusters with few Ar a second component in the Ne signal appears at higher binding energy, which is explained by formation of thicker Ne layers with surface states bound to other Ne atoms (green trace). Consequentially, the mixed ICD signal is quenched in panel b) by ICD to $\mathrm{Ne}^{+} \mathrm{Ne}^{+}$final states. Dotted lines mark the binding energies of Ne 2s surface [9] and interface states [32], the letter 'A' designates the atomic 2s peak. 
Figure 6: Intensity of electron pairs recorded after photoionization of a free $\langle N\rangle=45$ Ne cluster jet at a photon energy of $51.8 \mathrm{eV}$ [27]. The intensity of electron pairs detected in coincidence (panel b), with kinetic energy of the fast electron $\left(e_{1}\right)$ recorded on the vertical, and of the slow electron $\left(e_{2}\right)$ on the horizontal axis, allows to derive the energy spectrum of ICD (panel a) and the binding energy of the two-hole final states populated in the decay (panel d). The ICD spectrum was produced by summing up the intensity with $e_{1}$ kinetic energies between 3.15 and $4.15 \mathrm{eV}$, pertaining to a $2 \mathrm{~s}$ photoelectron, along the $e_{1}$ axis. The region is marked by two red bars in panel b. The final state spectrum is obtained by summing up the coincident signal along the lines of constant total energy, at a right angle to the main diagonal of panel b. The gray shaded region is the range of final state energies which is populated by ICD. Panel c: Coincident intensity summed up along the $e_{2}$ axis.

Figure 7: Intensity of electron pairs recorded in coincidence after photoionization of free water clusters $(\langle N\rangle=200)$ at a photon energy of $60 \mathrm{eV}[16]$. See Fig. 6 and text.

Figure 8: Sketch of the $\operatorname{ETMD}(3)$ process: 1 . An inner valence electron is ionized (top row), 2. the vacancy is filled by electron transfer from a neighbouring atom or molecule, and the released energy ionizes a third cluster constituent (middle row), 3. the system undergoes Coulomb explosion (bottom row) $[96,53]$. In the $\operatorname{ETMD}(2)$ process, both final state vacancies are created in the same consituent of type ' $\mathrm{B}$ '. 

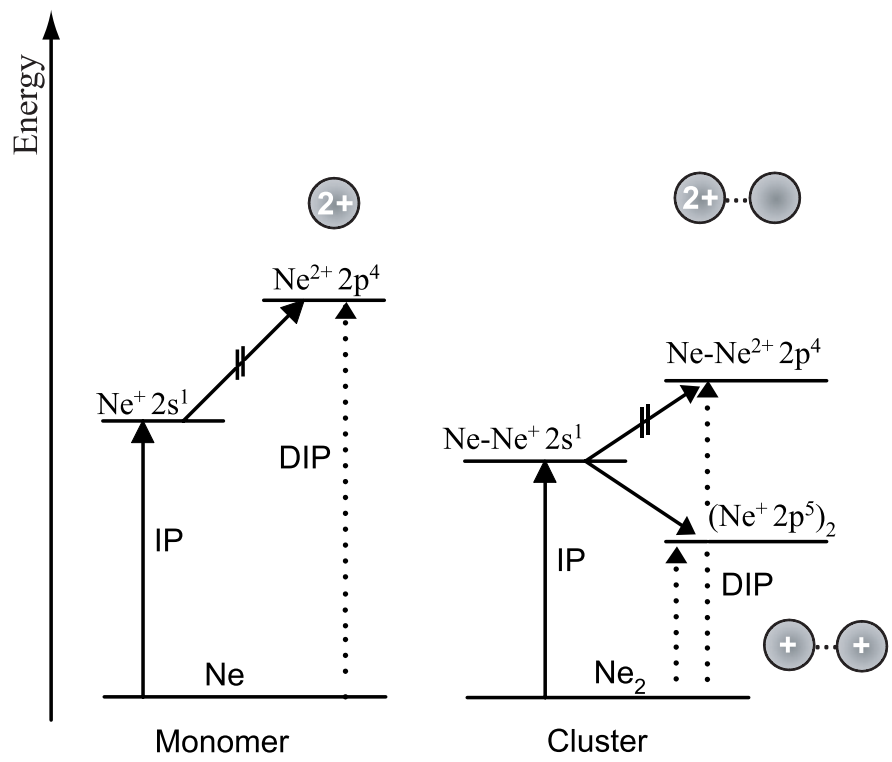

Figure 1: 


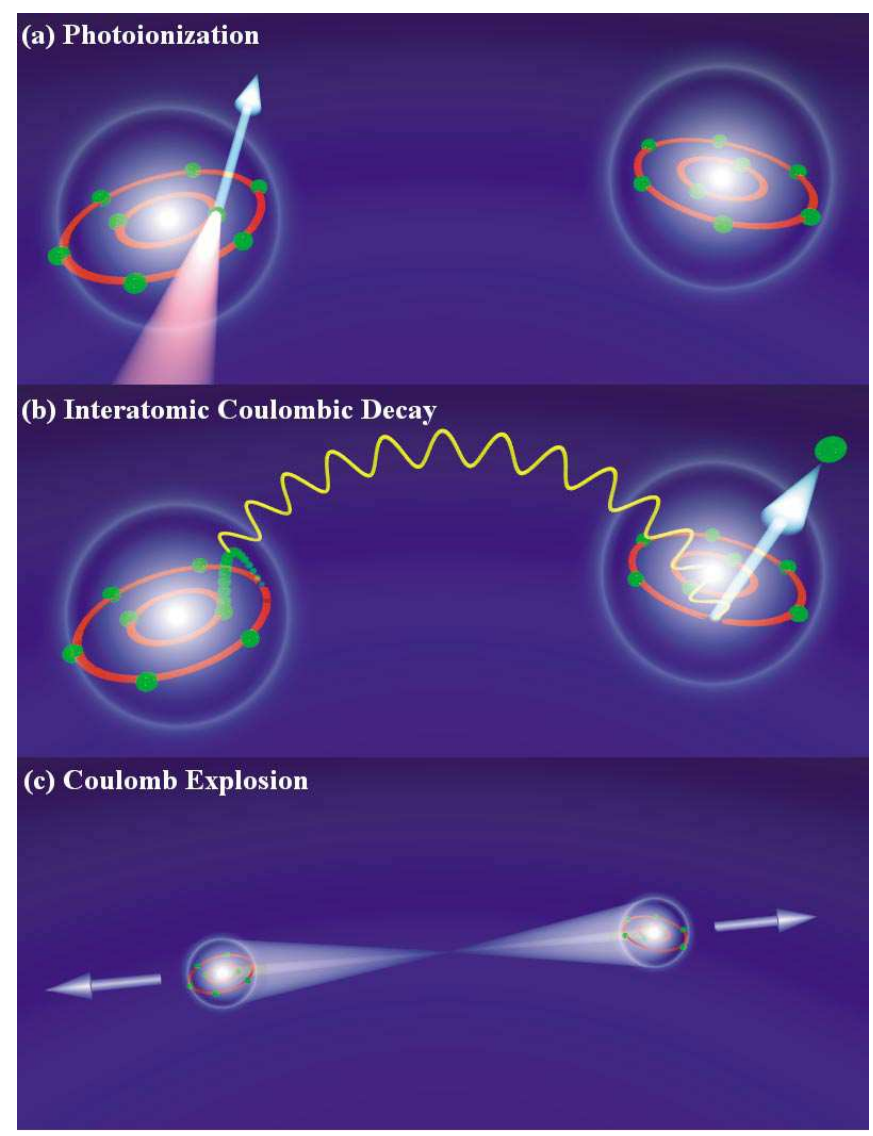

Figure 2:

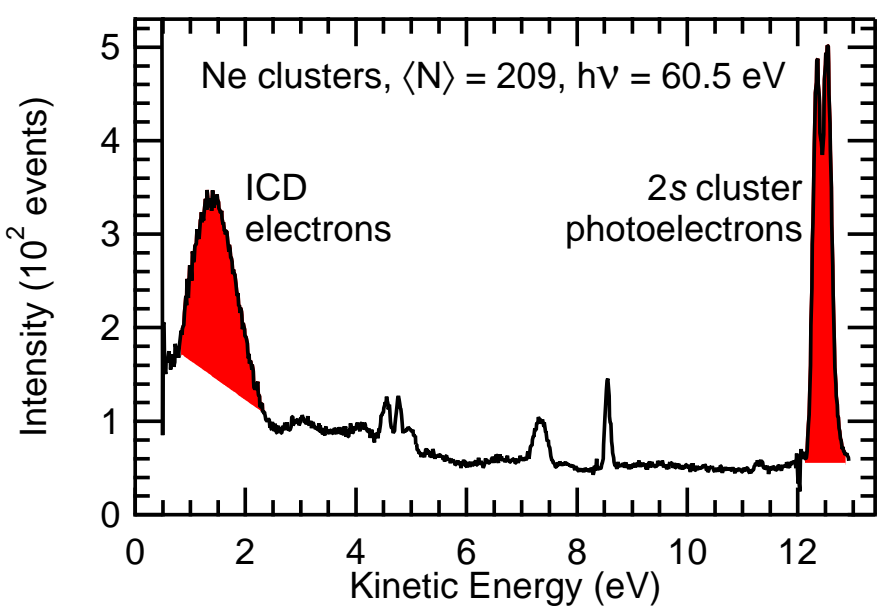

Figure 3: 


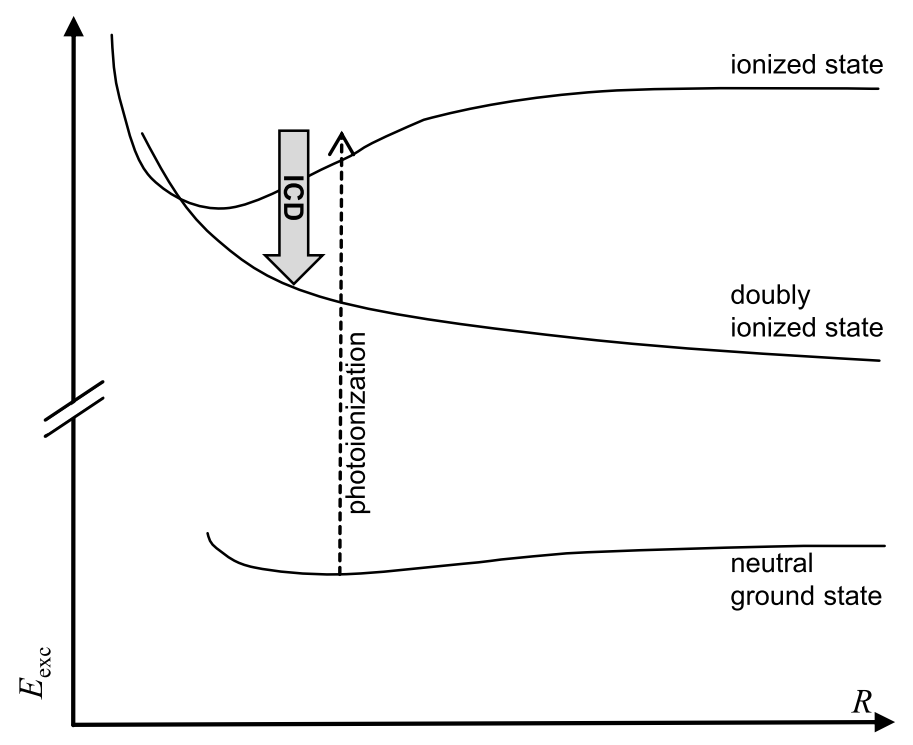

Figure 4:

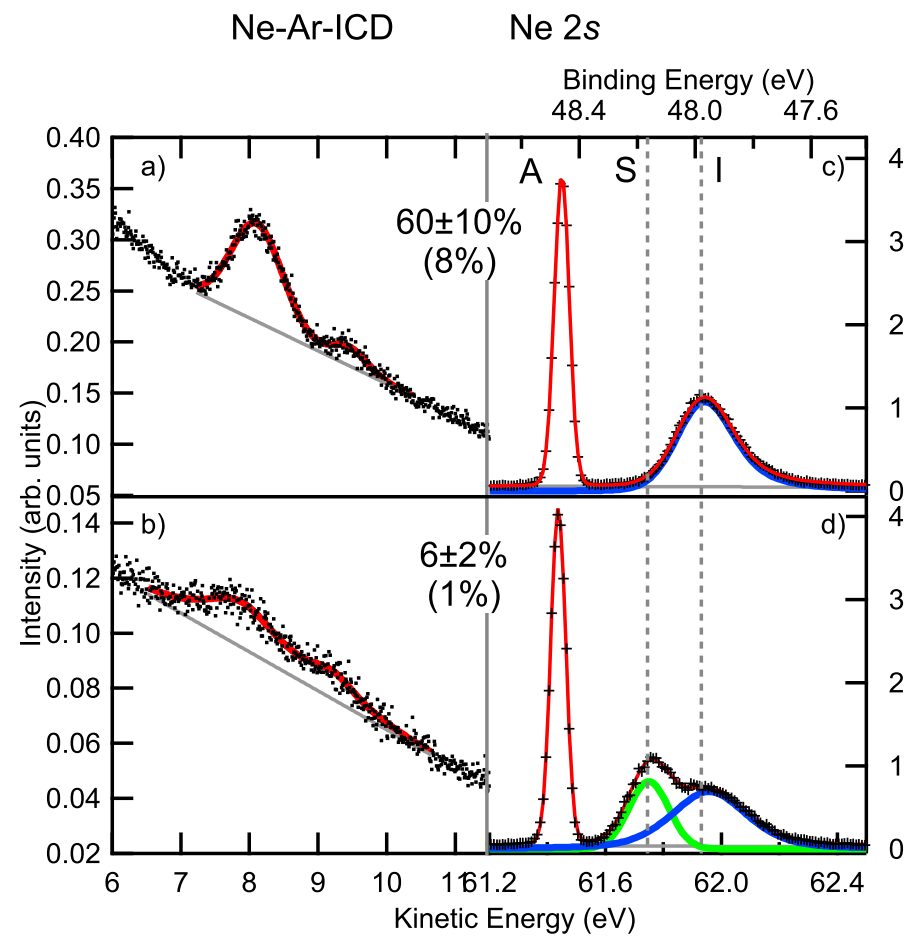

Figure 5: 

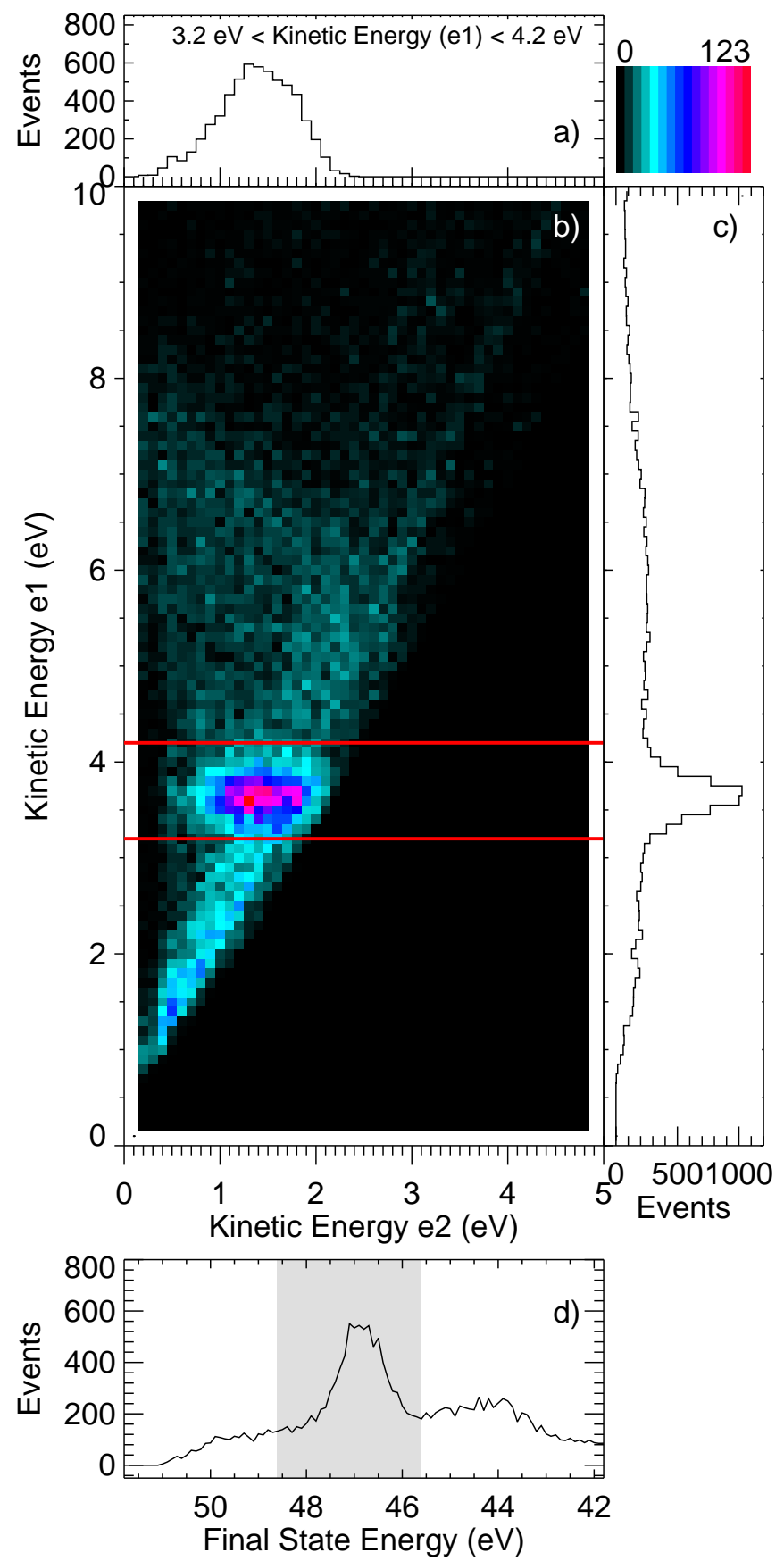

Figure 6: 


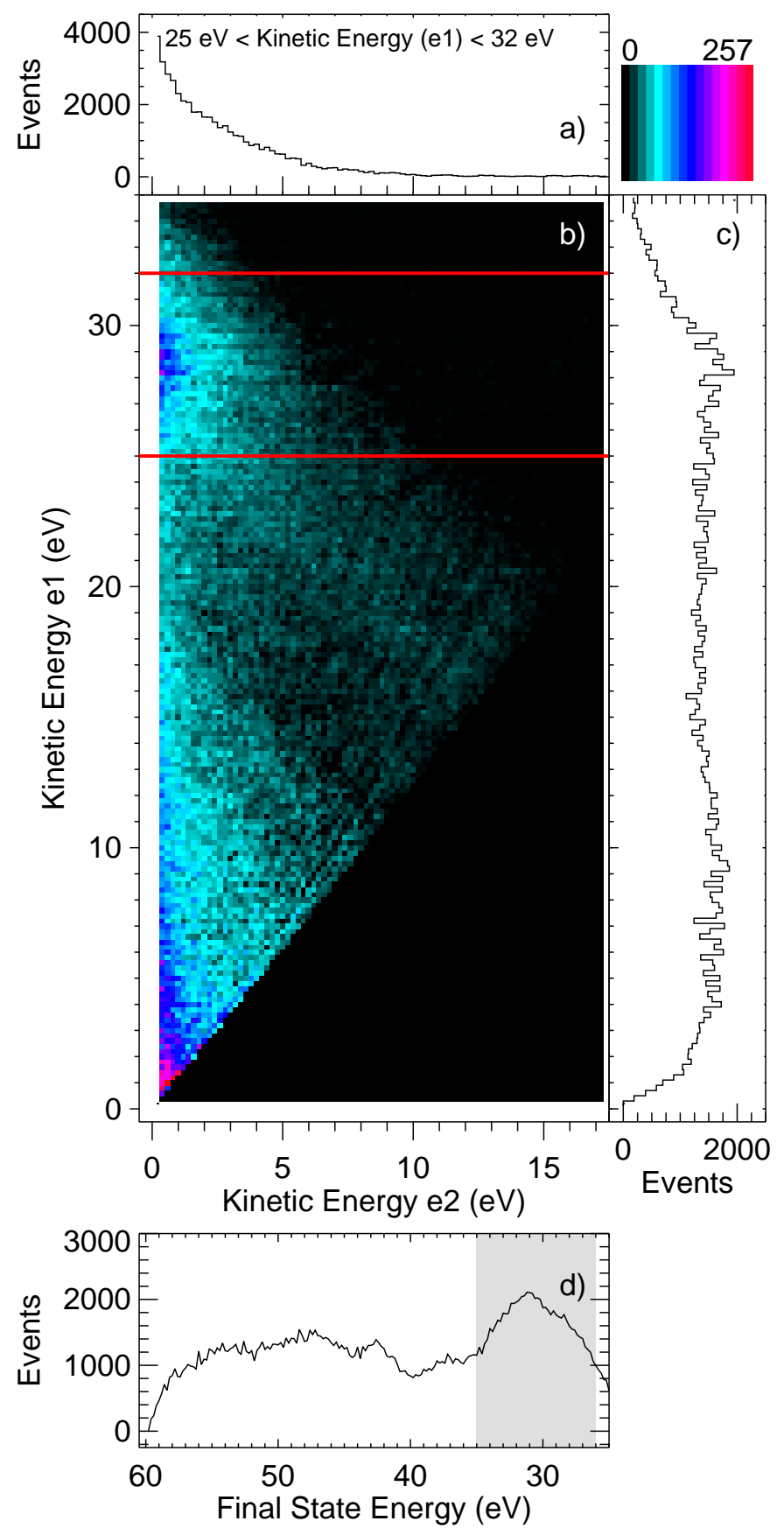

Figure 7: 

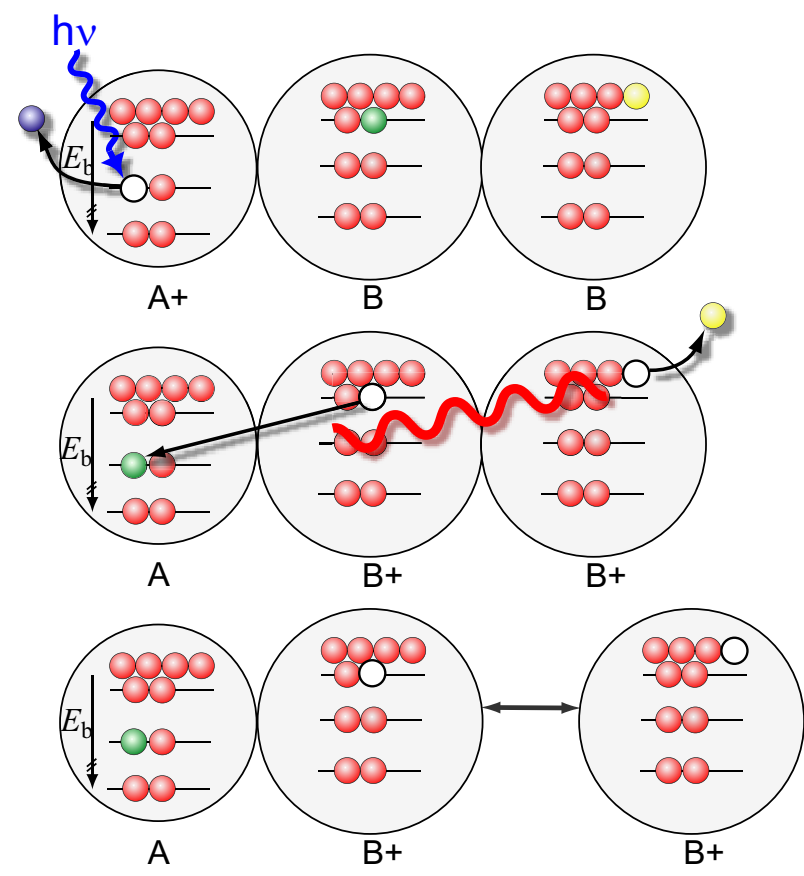

ETMD (3)

Figure 8: 